\title{
INPUT-TO-STATE STABILITY OF RATE-CONTROLLED BIOCHEMICAL NETWORKS*
}

\author{
MADALENA CHAVES ${ }^{\dagger}$
}

\begin{abstract}
In this paper, the study of the class of biochemical systems known as zero deficiency networks is extended to the case of time-varying kinetic parameters. We show that the resulting class of nonlinear systems with inputs satisfies a notion of input-to-state stability uniformly over a set of parameters. In particular, the input-to-state stability estimates allow us to characterize the robustness of zero deficiency networks with respect to perturbations in the parameters as well as study their stability when the reaction rates are controlled by an independent process.
\end{abstract}

Key words. stability, robustness, biochemical networks

AMS subject classifications. 93D25, 92C45

DOI. $10.1137 /$ S0363012903437964

1. Introduction. A biochemical network consists of the interactions among a certain number of species, according to a set of specified reactions that induce a dynamics for the species' concentrations. The time evolution of the species' concentrations is usually modeled by a system of differential equations together with a family of parameters that characterize the reaction rates. These parameters may depend on various external factors and stimuli such as temperature, the concentration of ligands/substrates, or the concentration of an enzyme which may be regulated by an independent dynamics.

Thus biological systems may, in many cases, be viewed as cascades of biochemical networks, where the output of the $i$ th level becomes the input to the $(i+1)$ th level of the cascade. This is indeed the structure of many intracellular signal transduction pathways, which are central to biological processes. For example, the binding of a ligand to a cell receptor triggers a sequence of biochemical reactions [12] that ultimately lead to a cell response (such as contraction, motility, or proliferation).

Each level in the cascade may be studied independently as a system with inputs, and in particular we will focus on the input-to-state stability properties of such system. We are also interested in studying the effect of small parameter perturbations (which may be due, for instance, to variations in the room temperature or other experimental setup problems) on the steady-state response of the system. The notion of a parameter robust system should reflect the idea that these small perturbations should not greatly affect the qualitative response and guarantee that the output error will also be small. In addition, the input-to-state stability properties of a system provide a framework for the analysis of the stability and convergence of cascades of systems (see, for instance, $[15,2]$ ).

A mathematical model for a certain family of biochemical networks, where the reactions satisfy the mass action kinetics principle, was introduced by Horn and Jackson in 1972 [9] and followed up by the work of Feinberg [6, 7, 8]. These authors

\footnotetext{
* Received by the editors November 25, 2003; accepted for publication (in revised form) April 5, 2005; published electronically September 12, 2005.

http://www.siam.org/journals/sicon/44-2/43796.html

${ }^{\dagger}$ Department of Mathematics, Rutgers University, 110 Frelinghuysen Rd., Piscataway, NJ 08854 (madalena@math.rutgers.edu). The author was supported in part by Fundação para a Ciência e a Tecnologia, by Fundação Calouste Gulbenkian, Portugal, and also in part by the BioMaPS Institute at Rutgers University, Aventis (Bridgewater, NJ) and AFOSR (grant F49620-01-1-0063).
} 
developed a rich and beautiful theory on these systems, also known in the literature as zero deficiency networks. This model accommodates a wide variety of significant biological systems, including many models for enzymatic mechanisms [14], a model for T-cell receptor signal transduction [13], and receptor-ligand interactions and Gprotein coupled receptor activity in cyclic signaling pathways $[3,12,19]$. The models of receptor-ligand interaction are of interest for biomedical applications as well as for drug design: the concentration-response curves associated with some of the basic models $[3,12]$ may also be analyzed in the context of these zero deficiency networks [5].

The zero deficiency networks may be characterized in terms of a strongly connected graph and the corresponding irreducible matrix, while the mass action kinetics property leads to nonlinear systems with polynomial vector fields. These are the systems we consider in this paper: for these systems, which exhibit multiple steady states, the state space may be viewed as a (disjoint) union of invariant manifolds (which are parallel translates of a given subspace of $\mathbb{R}^{n}$ ) so that to each of these invariant manifolds corresponds a distinct (globally) asymptotically stable steady state. The idea of invariant sets of the state space and the existence of steady states, and how these are affected by the external inputs or perturbations will be central to our analysis.

Recently [17], this class of nonlinear systems was studied from a control theory point of view, and the stability and other properties for the system with no inputs were further analyzed. In [17] a formalism is developed for dealing with this type of systems and several results are established which will be frequently referred to in the present work.

We first introduce the class of systems to be studied and recall some basic results (section 2). The definition and characterization of the notion of uniformly semiglobal input-to-state stable systems as well as the statement of the main results are given in section 3. The input-to-state stability estimates are established and the main theorems are proved in sections 5 and 6 . In section 4 we show the dependence of steady states of the system on its parameters: the unique steady state in each invariant manifold is an analytic function of the parameters. Section 7 summarizes the main contributions in this paper.

2. Some notation and previous results. Let $n \geq m$ be integers and let $x \in \mathbb{R}^{n}$. Introduce the positive orthant

$$
\mathbb{R}_{>0}^{n}=\left\{\left(x_{1}, \ldots, x_{n}\right) \in \mathbb{R}^{n}: x_{i}>0 \quad \forall i\right\}
$$

and the closed positive orthant

$$
\mathbb{R}_{\geq 0}^{n}=\left\{\left(x_{1}, \ldots, x_{n}\right) \in \mathbb{R}^{n}: x_{i} \geq 0 \quad \forall i\right\} .
$$

Assume given two matrices $A=\left(a_{i j}\right) \in \mathbb{R}_{\geq 0}^{m \times m}$ and $B \in \mathbb{N}_{0}^{n \times m}$, where the columns of $B$ are denoted by $b_{1}, \ldots, b_{m}$. In our model of biochemical reactions there are $n$ distinct species whose concentrations are given by $x=\left(x_{1}, \ldots, x_{n}\right)^{\prime}$ and $m$ complexes, each complex denoting a set of reactants or products in a reaction. The complexes are represented by the column vectors $b_{1}, \ldots, b_{m}$ with $b_{j}=\left(b_{1 j}, \ldots, b_{n j}\right)^{\prime}$ and $b_{l j} \neq 0$ if the species $l$ appears in the complex $j$. The matrix $A=\left(a_{i j}\right)$ is the matrix of the kinetic constants, and an entry $a_{i j} \neq 0$ means that complex $i$ is being produced from complex $j$.

The model for biochemical reaction networks of the Horn-Jackson-Feinberg zero 
deficiency type, with mass action kinetics, is as follows:

$$
\dot{x}=f_{A}(x):=\sum_{i=1}^{m} \sum_{j=1}^{m} a_{i j} x_{1}^{b_{1 j}} x_{2}^{b_{2 j}} \cdots x_{n}^{b_{n j}}\left(b_{i}-b_{j}\right) .
$$

Since the vector $x \in \mathbb{R}^{n}$ represents the concentration of each species involved in the reactions, we will be interested only in those trajectories that evolve in the positive orthant. In fact, it is easy to see that the positive orthant $\mathbb{R}_{>0}^{n}$ is a forward-invariant set for the system (2.1) (see also section 5). We have the following assumptions on $A$ and $B$.

(a) The matrix $B=\left(b_{1}, \ldots, b_{m}\right)$ has nonnegative integer entries; it has full column rank and none of its rows vanishes completely.

(b) The matrix $A=\left(a_{i j}\right)$ has nonnegative entries (without loss of generality, we assume that its diagonal entries are zero, since the corresponding terms would be of the form $\left.a_{i i} x_{1}^{b_{1 i}} \cdots x_{n}^{b_{n i}}\left(b_{i}-b_{i}\right) \equiv 0\right)$ and is assumed to be irreducible.

This last property is equivalent to saying that the incidence graph of $A$ is strongly connected, and it describes the following property of the network: there is a chemical pathway connecting every pair of complexes, but the pathway leading from $b_{i}$ to $b_{j}$ may be different from the pathway leading from $b_{j}$ back to $b_{i}$ (in other words, each individual chemical reaction is not necessarily reversible). Another equivalent definition of irreducibility says that there is an integer $k$ such that all the entries of the matrix $(A+I)^{k}$ are strictly positive (see [10]).

Example. The simplest model for the interaction of a cell surface receptor with a specific ligand is depicted as $R+L \leftrightharpoons C$. Here we have three species $(n=3)$ : receptor $(R)$, ligand $(L)$, and receptor-ligand product $(C)$. There are only two complexes $(m=2): R+L$ and $C$. Let $x_{1}, x_{2}$, and $x_{3}$ denote the concentrations of $R, L$, and $C$, respectively. Then the two columns of matrix $B$ are $b_{1}=(1,1,0)^{\prime}$ and $b_{2}=(0,0,1)^{\prime}$. The matrix $A$ is of size 2 and its nonzero elements are $a_{21}$ (the rate constant for $R+L \rightarrow C$ ) and $a_{12}$ (the rate constant for $C \rightarrow R+L$ ). It is easy to see that $\dot{x}_{1}=\dot{x}_{2}=-\dot{x}_{3}=-a_{21} x_{1} x_{2}+a_{12} x_{3}$. A network involving two distinct receptor conformations is shown in Figure 1.

Example. Another simple example is a dimer model, where the ligand binds to two receptors, according to the diagram $2 R+L \leftrightharpoons R+C_{1} \leftrightharpoons C_{2}$. In this case $n=4$ and $m=3$. Setting $x=\left(R, L, C_{1}, C_{2}\right)$, the matrix $B$ consists of the vectors $b_{1}=(2,1,0,0)^{\prime}, b_{2}=(1,0,1,0)^{\prime}$, and $b_{3}=(0,0,0,1)^{\prime}$. The nonnegative entries of the matrix $A$ are $a_{21}, a_{12}, a_{32}$, and $a_{23}$. Again, it is easy to see that properties (a) and (b) are satisfied. At any given time, the concentration of receptors is given by the equation $\dot{R}=-a_{21} R^{2} L-\left(a_{32}-a_{12}\right) R C_{1}+a_{23} C_{2}$, and the concentration of ligand is given by $\dot{L}=-a_{21} R^{2} L+a_{12} R C_{1}$. The concentrations of $C_{1}$ and $C_{2}$ are given by $\dot{C}_{1}=-\left(a_{12}+a_{32}\right) R C_{1}+a_{21} R^{2} L+a_{23} C_{2}$ and $\dot{C}_{2}=-a_{23} C_{2}+a_{32} R C_{1}$, respectively.

In this paper, we wish to study system (2.1) when the parameters $a_{i j}$ are allowed to be time variant. Values of the parameters should be such that, at each time instant, the matrix $A=\left(a_{i j}\right)$ is irreducible, so we consider the set of irreducible $m \times m$ matrices whose entries are nonnegative:

$$
\mathcal{A}_{\geq 0}=\left\{A \in \mathbb{R}^{m \times m}: A \geq 0 \text { and }(A+I)^{k}>0 \text { for some power } k\right\}
$$

(the inequality $A \geq 0$ (resp., $A>0$ ) means that every entry of the matrix on the left-hand side is nonnegative (resp., positive)). Let $|A|_{\text {ecl }}$ denote the matrix norm induced by the vector norm $|\cdot|$ (the Euclidean norm). Throughout this paper, we will 
define an input $u(\cdot)$ to be a piecewise locally Lipschitz function, with a finite number of discontinuities, that is, there exist $\ell \in \mathbb{N}$ and numbers $0=T_{0}<T_{1}<\cdots<T_{\ell}<$ $T_{\ell+1}=\infty$ such that the function $u$ is locally Lipschitz on each interval $\left(T_{i-1}, T_{i}\right)$ : for each $i=1, \ldots, \ell+1$ and each compact interval $J \subset\left(T_{i-1}, T_{i}\right)$, there exists $\kappa>0$ such that

$$
|u(t)-u(s)| \leq \kappa|t-s| \quad \forall s, t \in J .
$$

In addition, the mass action kinetics model may be generalized as in [17], so we will consider the system with inputs

$$
\dot{x}=f(x, u):=\sum_{i=1}^{m} \sum_{j=1}^{m} u_{i j} \theta_{1}\left(x_{1}\right)^{b_{1 j}} \theta_{2}\left(x_{2}\right)^{b_{2 j}} \cdots \theta_{n}\left(x_{n}\right)^{b_{n j}}\left(b_{i}-b_{j}\right),
$$

where the same assumptions on $B$ hold and each map $\theta_{i}: \mathbb{R} \rightarrow[0,+\infty)$ has the following properties:

(c) $\theta_{i}$ is real analytic;

(d) $\theta_{i}(0)=0$;

(e) $\int_{0}^{1}\left|\ln \theta_{i}(r)\right| d r<\infty$;

(f) its restriction to $\mathbb{R}_{\geq 0}$ is strictly increasing and onto the set $\left[0, \sigma_{i}\right)$, where $0<\sigma_{i} \leq+\infty$.

Before stating the last condition that the functions $\theta_{i}$ should satisfy, let us introduce the following vector functions:

$$
\rho^{[n]}(x)=\left(\ln \theta_{1}\left(x_{1}\right), \ldots, \ln \theta_{n}\left(x_{n}\right)\right)^{\prime} \quad \text { and } \quad \exp ^{[n]}(v)=\left(e^{v_{1}}, \ldots, e^{v_{n}}\right)^{\prime}
$$

defined on $\mathbb{R}_{>0}^{n}$ and $\mathbb{R}^{n}$, respectively. (From now on, we will drop the superscript $n$ of $\rho^{[n]}$ and $\exp ^{[n]}$, since its value is usually clear from the context.)

Each $\theta_{i}$ (restricted to $\mathbb{R}_{>0}$ ) is onto the set $\left(0, \sigma_{i}\right)$, so each function $\rho_{i}=\ln \theta_{i}$ (for the restriction of $\theta_{i}$ to $\left.\mathbb{R}_{>0}\right)$ is onto $\left(-\infty, \bar{\rho}_{i}\right)$ with $\bar{\rho}_{i}=\ln \sigma_{i}$. Since $\theta_{i}$ (restricted to $\left.\mathbb{R}_{\geq 0}\right)$ is strictly increasing, $\rho_{i}$ has an inverse function, which is onto $\mathbb{R}_{>0}: \rho_{i}^{-1}$ : $\left(-\infty, \bar{\rho}_{i}\right) \rightarrow \mathbb{R}_{>0}$. Each function $\theta_{i}$ should also satisfy

(g) for any given constant $p, \lim _{t \rightarrow \ln \sigma_{i}} \int_{a}^{t} \rho_{i}^{-1}(s) d s-p t=+\infty$ for any $a<\ln \sigma_{i}$. Note that, for any constant $p$, there exists $t_{0} \in\left(-\infty, \bar{\rho}_{i}\right)$ such that $\rho_{i}^{-1}(s)>p+1$ for all $s \geq t_{0}$. Therefore, when $\sigma_{i}=+\infty$, condition (g) always holds. The case of mass action kinetics corresponds to $\theta_{i}(r)=|r| \forall i$. Another example of interest is the case $\theta_{i}(r)=\frac{|r|}{k+|r|}$ with $k>0$. Even though condition (g) will not be used explicitly in this paper, it is necessary to prove some auxiliary results such as Lemma IV.1 in [17], which will be used in section 4 .

For the case of a constant matrix $A$, system (2.3) has been extensively studied. It takes the form

$$
\dot{x}=f(x, A):=f_{A}(x),
$$

and we next recall some results already established about this system, which are given in $[6,7,8,9]$ (for the particular case of mass action kinetics), and can also be found in [17] (for the general case).

For any two vectors $a, b \in \mathbb{R}^{n}$, let $\langle a, b\rangle$ denote their dot product. Define the stoichiometric space

$$
\mathcal{D}=\operatorname{span}\left\{b_{i}-b_{j}: i, j=1, \ldots, m\right\}
$$


and also consider its orthogonal space

$$
\mathcal{D}^{\perp}:=\left\{v \in \mathbb{R}^{n}:\langle v, d\rangle=0 \quad \forall d \in \mathcal{D}\right\} .
$$

Then, for any $v \in \mathcal{D}^{\perp}$, notice that

$$
\left\langle f_{A}, v\right\rangle=\sum_{i=1}^{m} \sum_{j=1}^{m} a_{i j} x_{1}^{b_{1 j}} \cdots x_{n}^{b_{n j}}\left\langle\left(b_{i}-b_{j}\right), v\right\rangle \equiv 0
$$

by the definition of $v$. Hence $\langle x(t), v\rangle=$ constant $=\langle x(0), v\rangle$, and we have

$$
\langle x(t)-x(0), v\rangle=0 \quad \forall v \in \mathcal{D}^{\perp} \Leftrightarrow x(t)-x(0) \in \mathcal{D} \Leftrightarrow x(t) \in x(0)+\mathcal{D} .
$$

Therefore, the parallel translates of the stoichiometric space, $p+\mathcal{D}$ with $p \in \mathbb{R}_{>0}^{n}$, define invariant manifolds for the system $\dot{x}=f_{A}(x)$. For each $p \in \mathbb{R}_{>0}^{n}$, we will define a positive class of system (2.4) to be

$$
\mathcal{S}:=(p+\mathcal{D}) \cap \mathbb{R}_{\geq 0}^{n}=\{p+d: d \in \mathcal{D}\} \cap \mathbb{R}_{\geq 0}^{n} .
$$

(If $\mathcal{S} \subset \partial \mathbb{R}_{\geq 0}^{n}$, then we do not consider such $\mathcal{S}$ as a positive class. In this work, we are not concerned with trajectories that evolve on the boundary of the positive orthant.) Note that the positive classes do not depend on the matrix $A$, only on $B$.

The equilibria of system (2.4) may be divided into boundary equilibria, $E_{0}=\{x \in$ $\left.\partial \mathbb{R}_{\geq 0}^{n}: f_{A}(x)=0\right\}$, and positive equilibria, $E_{A,+}=\left\{x \in \mathbb{R}_{>0}^{n}: f_{A}(x)=0\right\}$.

From [17, Proposition VI.3] we know that $E_{0}$ depends only on the matrix $B$ and not on $A$ (however, the elements in $E_{A,+}$ may depend on the matrix $A$ ). Throughout this paper we will assume that no boundary equilibria exist in any positive class, i.e.,

$$
\mathcal{S} \cap E_{0}=\emptyset \quad \text { for each positive class } \mathcal{S} .
$$

Under these conditions we have the following result from [17] and also [6].

TheOrem 2.1. Consider system (2.4) and assume that condition (2.6) holds. Then, for each positive class $\mathcal{S}$ there exists a (unique) state $\bar{x}=\bar{x}_{\mathcal{S}} \in \mathbb{R}_{>0}^{n}$ which is a globally asymptotically stable point relative to $\mathcal{S}$, i.e., for each $x_{0} \in \mathcal{S}$, the solution of $\dot{x}=f_{A}(x), x(0)=x_{0}$, is defined $\forall t \geq 0$, and $x(t) \rightarrow \bar{x}$ as $t \rightarrow \infty$, and $\forall \varepsilon>0$ there exists $\delta>0$ such that if $\left|\bar{x}-x_{0}\right|<\delta$, then $|\bar{x}-x(t)|<\varepsilon \forall t>0$.

Throughout this paper, we will assume that the matrix $B$ (which defines the complexes that form the network) is fixed. Each matrix $A \in \mathcal{A}_{\geq 0}$ characterizes a system of the form (2.4), and we will let $x\left(t, x_{0}, A\right)$ denote the solution of the differential equation $\dot{x}=f_{A}(x)$ at time $t$, when the initial condition is $x(0)=x_{0} \in$ $\mathbb{R}_{>0}^{n}$. Then, from Theorem 2.1, it follows that each trajectory $x\left(\cdot, x_{0}, A\right)$ converges to the positive equilibrium in the same class as $x_{0}$. So we define $\bar{x}\left(x_{0}, A\right)$ to be the unique equilibrium in the same class as $x_{0}$, and thus we may also write

$$
E_{A,+}=\left\{\bar{x}\left(x_{0}, A\right): x_{0} \in \mathbb{R}_{>0}^{n}\right\}
$$

and introduce the set of all such positive equilibrium points:

$$
\mathcal{E}=\bigcup_{A \in \mathcal{A} \geq 0} E_{A,+}
$$

In section 4 we will show that, as a map from $\mathbb{R}_{>0}^{n} \times \mathcal{A}_{\geq 0}$ to $\mathbb{R}^{n}, \bar{x}(\cdot, \cdot)$ is a real analytic function. 


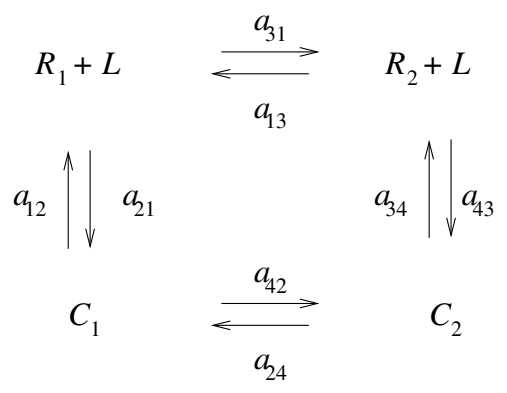

FIG. 1. A receptor-ligand network.

2.1. An example. As a motivation for our theoretical results, we will discuss a nominal example. The biochemical network depicted in Figure 1 is a basic model for receptor-ligand interactions at the cell surface [3, 12]. The ligand is denoted by $L$, two cell receptor conformations are denoted by $R_{1}$ and $R_{2}$, and the respective receptor-ligand complexes are denoted by $C_{1}$ and $C_{2}$. These constitute the $n=5$ individual species, $X=\left(R_{1}, R_{2}, L, C_{1}, C_{2}\right)^{\prime}$. There are $m=4$ complexes, $R_{1}+L, C_{1}$, $R_{2}+L$, and $C_{2}$. This model may be characterized by the matrices

$$
A=\left(\begin{array}{cccc}
0 & a_{12} & a_{13} & 0 \\
a_{21} & 0 & 0 & a_{24} \\
a_{31} & 0 & 0 & a_{34} \\
0 & a_{42} & a_{43} & 0
\end{array}\right), \quad B=\left(\begin{array}{cccc}
1 & 0 & 0 & 0 \\
0 & 0 & 1 & 0 \\
1 & 0 & 1 & 0 \\
0 & 1 & 0 & 0 \\
0 & 0 & 0 & 1
\end{array}\right),
$$

where $A$ is clearly irreducible. Under mass action kinetics, the dynamics of the system is

$$
\begin{aligned}
\dot{R}_{1} & =-\left(a_{21}+a_{31}\right) R_{1} L+a_{12} C_{1}+a_{13} R_{2} L, \\
\dot{R}_{2} & =-\left(a_{13}+a_{43}\right) R_{2} L+a_{31} R_{1} L+a_{34} C_{2}, \\
\dot{L} & =-a_{21} R_{1} L-a_{43} R_{2} L+a_{12} C_{1}+a_{34} C_{2}, \\
\dot{C}_{1} & =-\left(a_{12}+a_{42}\right) C_{1}+a_{21} R_{1} L+a_{24} C_{2}, \\
\dot{C}_{2} & =-\left(a_{34}+a_{24}\right) C_{2}+a_{42} C_{1}+a_{43} R_{2} L .
\end{aligned}
$$

The stoichiometric space is given by

$$
\begin{aligned}
\mathcal{D} & =\operatorname{span}\left\{b_{i}-b_{j}: i, j=1, \ldots, 4\right\} \\
& =\operatorname{span}\left\{(1,0,1,-1,0)^{\prime},(1,-1,0,0,0)^{\prime},(1,0,1,0,-1)^{\prime}\right\},
\end{aligned}
$$

and the positive classes are thus characterized by a pair of positive constants $\left(\alpha_{1}, \alpha_{2}\right)$,

$$
L+C_{1}+C_{2}=\alpha_{1}, \quad R_{1}+R_{2}+C_{1}+C_{2}=\alpha_{2},
$$

and, incidentally, note that the classes reflect the conservation of the total amount of ligand and the total amount of cell receptors in the system. The boundary equilibria set is given by

$$
E_{0}=\left\{\left(r_{1}, r_{2}, 0,0,0\right)^{\prime},(0,0, l, 0,0)^{\prime}: r_{1}, r_{2}, l \in[0,+\infty)\right\},
$$

and it is easy to see that each of these equilibrium points implies either $\alpha_{1}=0$ or $\alpha_{2}=0$, which do not define a positive class. Therefore, the "no boundary equilibria" assumption (2.6) holds. 
In this receptor-ligand model, the kinetic parameters are assumed to be fixed. A rough numerical estimate of the effect of perturbations on the steady-states shows that, for a sufficiently large (and fixed) $T$,

$$
\left|x\left(T, x_{0}, A\right)-x\left(T, x_{0}, A_{0}\right)\right| \lesssim 0.15\left|A-A_{0}\right|_{\mathrm{ecl}},
$$

suggesting that the system is indeed parameter-robust and that, moreover, the error is not amplified. Figure 2 shows the effect on the trajectories of the system of random perturbations in the kinetic constants, while Figure 3 justifies estimate (2.8). In this figure, each point - corresponds to the mean square error at steady state for a given error in the kinetic constant $\left(\left|A-A_{0}\right|_{\text {ecl }}\right)$. To obtain Figure 3 , for each $p \in$ $\{10,20,30,40,50,60\}$, system $(2.7)$ was simulated 20 times for the same fixed initial condition $\left(x_{0}\right)$, with its kinetic constants randomly perturbed within $p \%$, i.e.,

$$
a_{i j}=\left(1+\tilde{p}_{i j}\right) a_{i j}^{0} \quad \text { with } \quad \tilde{p}_{i j} \in[-p / 100, p / 100] .
$$

For each simulation, the norms $\left|A-A_{0}\right|_{\text {ecl }}$ and $\left|x\left(T, x_{0}, A\right)-x\left(T, x_{0}, A_{0}\right)\right|$, for a sufficiently large $T$, were computed, and a point - was plotted. An average of the values $\left|x\left(T, x_{0}, A\right)-x\left(T, x_{0}, A_{0}\right)\right|$ over intervals $\left|A-A_{0}\right|_{\text {ecl }} \in\left[d_{0}, d_{1}\right]$ of length 0.16 was also computed, resulting in the open squares $(\square)$. The solid line represents the best linear fit to these average points with a slope of 0.05 . Finally, notice that mostly all points are below the dash-dotted line, that is, they satisfy estimate $(2.8)$.

For both figures, the initial condition was set to $x_{0}=(7,2,15,0.5,0.5)^{\prime}$ corresponding to a common situation where the amount of ligand is larger than the total amount of receptors, and there are practically no receptor-ligand complexes formed at the beginning of the reaction. The (ideal) values of the parameters were set to

$$
A_{0}=\left(\begin{array}{cccc}
0 & 0.25 & 0.8 & 0 \\
2.7 & 0 & 0 & 0.45 \\
0.9 & 0 & 0 & 0.25 \\
0 & 0.55 & 2.5 & 0
\end{array}\right)
$$

3. Input-to-state stability and robustness. We wish to study the system with inputs (2.3) and establish general estimates that reflect the stability result obtained numerically for the example in (2.8). To do this, we start by defining appropriate input-to-state stability notions. An important observation on the system is that positive classes are invariant not only under constant inputs but also under any time-variant input map with $u(t) \in \mathcal{A}_{\geq 0} \forall t$. This follows from the fact that the matrix $B$ (and hence also the stoichiometric space and its orthogonal space $\mathcal{D}^{\perp}$ ) is fixed, and also from (2.5). Indeed, let $\mathcal{S}_{\bar{x}}$ be any class (recall that each class may be characterized by a positive equilibrium $\bar{x} \in \mathcal{E}$ ). Then, for each initial condition $x_{0} \in \mathcal{S}_{\bar{x}}$ and input map $u(\cdot)$, the trajectory of system (2.3) evolves in this positive class for all times. Thus, any input-to-state stability estimates only need to hold in that class.

3.1. Definition of input-to-state stability in each invariant subspace. The input-to-state stability notion introduced in Definition 3.2 follows the ideas and the concept of input-to-state stability (ISS) first established in [15] (and see also the notion of input-output-to-state stability introduced in [11]). With the goal of analyzing positive systems, the main difference in our definition of ISS is the introduction of a condition on the completeness of the system with respect to positive states (i.e., those states with all coordinates in the strictly positive half-line). This condition 

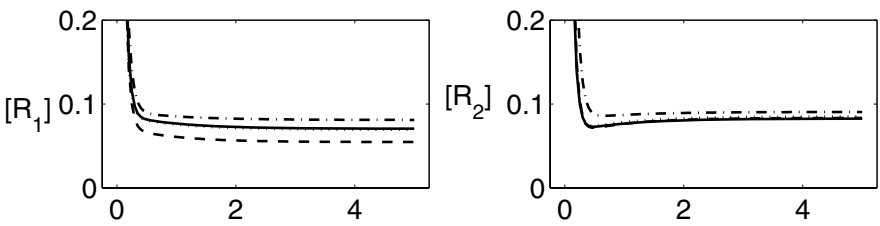

[L]
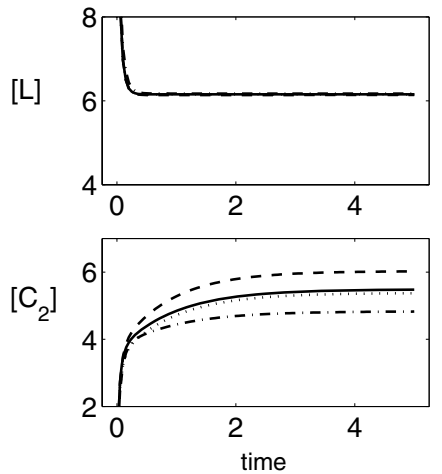
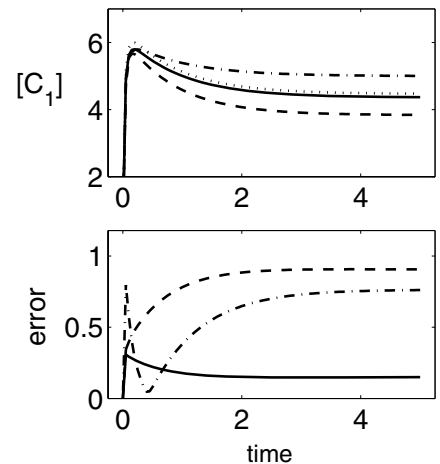

FIG. 2. The dotted lines represent the trajectory of the system $\dot{x}=f_{A_{0}}(x)$ ( $A_{0}$ as indicated in the text), while the solid, dashed, and dash-dotted lines represent the trajectories of $\dot{x}=f_{A}(x)$ with the entries of $A$ randomly chosen within, respectively, $10 \%, 20 \%$, and $30 \%$ of the (nonzero) entries of $A_{0}$. The error is computed as the norm $\left|x\left(t, x_{0}, A_{0}\right)-x\left(t, x_{0}, A\right)\right|$.

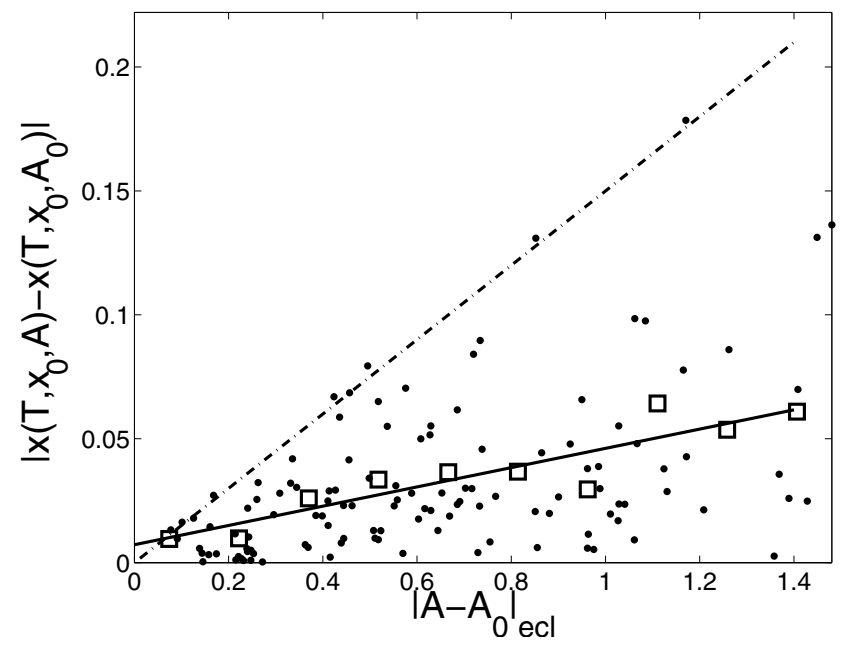

FIG. 3. The error in the steady states due to random perturbations in the kinetic constants $A_{0}$ (see explanation in the text).

plays an important part in the subsequent characterization of the ISS property in terms of an ISS-Lyapunov function: such a function need only be defined on $\mathbb{R}_{\geq 0}^{n}$ and differentiable on the strictly positive orthant, and it is not required to satisfy a decrease condition except at positive vectors, as stated in Definition 3.3 (in the original characterization, the ISS-Lyapunov function was defined in $\mathbb{R}^{n}$ ).

In addition, our notion of ISS is formulated as a semiglobal property, in the sense that the input-to-state estimates only hold while the trajectories remain in some preestablished compact set (see also [4]). And it is a uniform property, in the sense 
that the same functions provide input-to-state estimates for all trajectories evolving in $\cup_{\bar{x} \in P} \mathcal{S}_{\bar{x}}$, where $P$ is a compact subset of $\mathcal{E}$.

We first recall some standard notions that will be used in establishing estimates: a function $\alpha: \mathbb{R}_{\geq 0} \rightarrow \mathbb{R}_{\geq 0}$ is said to be of class $\mathcal{K}$ if it is continuous, strictly increasing, and $\alpha(0)=0$. The function $\alpha$ is said to be of class $\mathcal{K}_{\infty}$ if it is of class $\mathcal{K}$ and in addition $\alpha(r) \rightarrow+\infty$ as $r \rightarrow+\infty$. A function $\beta: \mathbb{R}_{\geq 0} \times \mathbb{R}_{\geq 0} \rightarrow \mathbb{R}_{\geq 0}$ is said to be of class $\mathcal{K} \mathcal{L}$ if, for each fixed $t, \beta(\cdot, t)$ is of class $\mathcal{K}$ and for each fixed $r, \beta(r, \cdot)$ is strictly decreasing with $\beta(r, t) \rightarrow 0$ as $t \rightarrow+\infty$.

For the following definitions, let the system $\dot{x}=f(x, u)$ evolve on a state space $\mathcal{X}$ which is an open subset of $\mathbb{R}^{n}$ containing $\mathbb{R}_{>0}^{n}$. Let $\mathbb{U}$ be a subset of $\mathcal{A}_{\geq 0}$, and let $A_{0} \in \mathbb{U}$. For any $\bar{x}_{0} \in \mathbb{R}_{>0}^{n}$, let $\mathcal{S}_{\bar{x}_{0}}$ represent any invariant set for the system $\dot{x}=f(x, u)\left(\right.$ with $\left.\bar{x}_{0} \in \mathcal{S}_{\bar{x}_{0}}\right)$. Let

$$
\left\|u-A_{0}\right\|=\operatorname{ess} \cdot \sup .\left\{\left|u(t)-A_{0}\right|_{\mathrm{ecl}}: t \in[0,+\infty)\right\} .
$$

Definition 3.1. A system $\dot{x}=f(x, u)$, with input-value set $\mathbb{U}$, is $\mathbb{R}_{>0}^{n}$-forward invariant if, for each initial state $x(0) \in \mathbb{R}_{>0}^{n}$ and each $\mathbb{U}$-valued input $u(\cdot)$, the corresponding maximal solution of $\dot{x}=f(x, u)$ as a differential equation in $\mathcal{X}$, which is defined on an interval $J_{x(0), u}=\left[0, t_{m a x}\right)$, has values $x(t) \in \mathbb{R}_{>0}^{n} \forall t \in J_{x(0), u}$. The system is $\mathbb{R}_{>0}^{n}$-forward complete if it is $\mathbb{R}_{>0}^{n}$-forward invariant and, for each $x(0) \in \mathbb{R}_{>0}^{n}$ and $\mathbb{U}$-valued input $u(\cdot), J_{x(0), u}=[0,+\infty)$.

Definition 3.2. A system $\dot{x}=f(x, u)$ is uniformly semiglobal input-to-state stable with input-value set $\mathbb{U}$ if

(i) the system is $\mathbb{R}_{>0}^{n}$-forward complete, and

(ii) for every compact set $P \subset \mathcal{E}$ and every compact set $F \subset \mathbb{R}_{\geq 0}^{n}$ containing $P$, there exist functions $\beta=\beta_{P, F}$ of class $\mathcal{K} \mathcal{L}$ and $\varphi=\varphi_{P, F}$ of class $\mathcal{K}_{\infty}$ such that, for every $\bar{x}_{0} \in P \cap E_{A_{0},+}$ for some $A_{0} \in \mathbb{U}$,

$$
\left|x(t)-\bar{x}_{0}\right| \leq \beta\left(\left|x_{0}-\bar{x}_{0}\right|, t\right)+\varphi\left(\left\|u-A_{0}\right\|\right)
$$

for each $\mathbb{U}$-valued input $u(\cdot)$ and every initial condition $x_{0} \in F \cap \mathcal{S}_{\bar{x}_{0}}{ }^{1}$ and $\forall t \geq 0$ such that $x(s) \in F \forall s \in[0, t]$.

If the functions $\beta, \varphi$ given in (ii) may be chosen independently of the compact $F$, then the system is uniformly input-to-state stable with input-value set $\mathbb{U}$.

Definition 3.3. A continuous function $V: \mathbb{R}_{\geq 0}^{n} \rightarrow \mathbb{R}_{\geq 0}$ is a uniformly semiglobal ISS-Lyapunov function for the system $\dot{x}=f(x, u)$ with input-value set $\mathbb{U}$ if

(i) the restriction of $V$ to $\mathbb{R}_{>0}^{n}$ is continuously differentiable; so that

(ii) for every compact $P \subset \mathcal{E}$, there exist functions $\nu_{1}=\nu_{1, P}, \nu_{2}=\nu_{2, P} \in \mathcal{K}_{\infty}$,

$$
\nu_{1}\left(\left|x-\bar{x}_{0}\right|\right) \leq V(x) \leq \nu_{2}\left(\left|x-\bar{x}_{0}\right|\right)
$$

for each $\bar{x}_{0} \in P$ and $\forall x \in \mathbb{R}_{>0}^{n}$;

(iii) for every compact set $P \subset \mathcal{E}$ and every compact set $F \subset \mathbb{R}_{>0}^{n}$ containing $P$, there exist functions $\alpha=\alpha_{P, F}, \gamma=\gamma_{P, F} \in \mathcal{K}_{\infty}$ such that, for every $\bar{x}_{0} \in P \cap E_{A_{0},+}$ for some $A_{0} \in \mathbb{U}$,

$$
\nabla V(x) f(x, u) \leq-\alpha\left(\left|x-\bar{x}_{0}\right|\right)+\gamma\left(\left|u-A_{0}\right|\right)
$$

for every $u \in \mathbb{U}$ and every $x \in F \cap \mathcal{S}_{\bar{x}_{0}} \cap \mathbb{R}_{>0}^{n}$.

\footnotetext{
${ }^{1}$ Since $P \subset F$, the intersection $F \cap \mathcal{S}_{\bar{x}_{0}}$ is nonempty, containing at least the point $\bar{x}_{0}$.
} 
If the functions $\alpha, \gamma$ given in (iii) may be chosen independently of the compact $F \subset \mathbb{R}_{\geq 0}^{n}$, then the function $V$ is a uniformly ISS-Lyapunov function for the system.

We next state without proof that the existence of an ISS-Lyapunov function implies that the system is input-to-state stable (in the sense of the previous definitions). The proof of the lemma is very similar to what is done in the case of the usual definition of an ISS system and follows closely the argument given in [18]. One should keep in mind that the Lyapunov function is differentiable only on the positive orthant, and that the trajectories evolve in invariant classes. (For a similar adaptation of the proof given in [18], see also [4].)

LEMma 3.4. Consider an $\mathbb{R}_{>0}^{n}$-forward complete system $\dot{x}=f(x, u)$ with inputvalue set $\mathbb{U}$. Suppose that there is a uniformly (semiglobal) ISS-Lyapunov function $V$ for the system. Then, the system is uniformly (semiglobal) input-to-state stable with input-value set $\mathbb{U}$.

3.2. Main results. As already mentioned, the work of Horn and Jackson, and Feinberg $[6,7,8,9]$ on zero deficiency biochemical networks considers only constant kinetic parameters. This is also the case in the recent work developed in $[17,4]$. In other words, so far the focus has been on systems (2.3) with constant inputs, $u_{i j}(t) \equiv a_{i j}$. In this paper, our goal is to study the stability and robustness of zero deficiency networks under time-varying parameters. In order to establish our stability results a "lower bound" on the parameters will be assumed, that is, given any $\varepsilon>0$, we consider the input-value set to be the following subset of $\mathcal{A}_{\geq 0}$ :

$$
\mathbb{U}_{\varepsilon}=\left\{A \in \mathcal{A}_{\geq 0}: a_{i j} \geq \varepsilon \text { or } a_{i j}=0\right\} .
$$

Note, however, that no upper bound on the values of $a_{i j}$ is required. In addition, recall that the input maps satisfy the regularity condition (2.2). So, we define

$$
\mathcal{W}:=\left\{w:[0,+\infty) \rightarrow \mathbb{U}_{\varepsilon} \mid w \text { is a piecewise locally Lipschitz function }\right\}
$$

The main results state that, first, system (2.3) is uniformly semiglobal ISS, and second, if (2.3) is mass-conservative, then it is also uniformly ISS. The proofs of the theorems are presented in section 6: the ISS properties are established by showing that the system admits a uniformly (semiglobal) ISS-Lyapunov function (section 5.1).

TheOREM 3.5. System (2.3) with the state space $\mathcal{X}=\mathbb{R}^{n}$, restricted to taking input maps $w \in \mathcal{W}$, is uniformly semiglobal ISS with input-value set $\mathbb{U}_{\varepsilon}$.

TheOREm 3.6. Suppose that system (2.3) with state space $\mathcal{X}=\mathbb{R}^{n}$ satisfies

$$
\exists v \in \mathbb{R}_{>0}^{n}, \quad v \cdot f(x, u)=0 \quad \forall x \in \mathcal{X} \quad \forall u \in \mathcal{A}_{\geq 0} .
$$

Then the system, when restricted to input maps $w \in \mathcal{W}$, is uniformly ISS with inputvalue set $\mathbb{U}_{\varepsilon}$.

We would like to point out that, in the particular case of the constant input $u(t) \equiv A_{0}$, Theorem 3.6 recovers the global stability result of Theorem 2.1 for massconservative systems. In fact, establishing that a given system is uniformly inputto-state stable with input-value set $\mathbb{U}_{\varepsilon}$ (appropriately chosen) provides an alternative proof of Theorem 2.1. Furthermore, in the case when the input consists of small perturbations around a desired value $A_{0}$, for instance, $u(t)=A_{0}+\delta(t)$, uniform (global) ISS implies robustness of the system with respect to $A_{0}$. In other words, if $\|\delta\| \leq \delta_{0}$, then we expect the difference between the desired and perturbed steady states of the system to satisfy $\left|\bar{x}-\bar{x}_{0}\right| \lesssim \varphi(\|\delta\|) \leq \varphi\left(\delta_{0}\right)$ (see also the example discussed in section 2.1). 
Remark. Condition (3.4) is satisfied by many biochemical systems; in particular, it is satisfied by mass-conservative systems, whose trajectories are a priori constrained to move in a compact subset of $\mathbb{R}_{\geq 0}^{n}$. A system of the form (2.3) is mass-conservative if and only if

$$
v=\sum_{i=1}^{n-m+1} v_{i} \in \mathbb{R}_{>0}^{n}
$$

where $\left\{v_{1}, \ldots, v_{n-m+1}\right\}$ is a basis of the space $\mathcal{D}^{\perp}$. Recall that, by definition of the invariant classes, for each $v_{i}$ there exists a positive constant $\alpha_{i}$ such that $\left\langle v_{i}, x(t)\right\rangle=$ $\alpha_{i}, \forall t \in J$. Then $\langle v, x(t)\rangle=\sum \alpha_{i}$ for every $t$, and in those cases where $v$ has all coordinates positive, we immediately have that $x(t)$ evolves in a compact subset of $\mathbb{R}_{\geq 0}^{n}$ and hence a compact subset of the state space. The example discussed in section 2.1 is mass-conservative with $v=(1,1,1,2,2)^{\prime}$.

Remark. One of the main assumptions in the model (2.3) is that, for all times $t$, the incidence graph of the matrix $u(t)$ is strongly connected or, equivalently, $u(t)$ is irreducible; hence the input $u$ is only allowed to take values in $\mathcal{A}_{\geq 0}$. However, in some ways, the structure of the network may be modified, i.e., new reactions may be added and existing reactions may be removed, provided that the irreducibility of the matrix $u(t)$ is not violated at any time $t$. This is guaranteed by requiring that $u \in \mathbb{U}_{\varepsilon}$.

As discussed above, Theorems 3.6 and 3.5 hold for input maps that are piecewise locally Lipschitz. These include many of the typical biological inputs such as piecewise constant, periodic, or exponentially decaying signals.

Example. As mentioned in the introduction, changes in the temperature induce changes in the value of the reaction rate constants. These changes are given by the Arrhenius law [1]:

$$
k=k(T):=F_{a} e^{-\frac{E_{a}}{R T}},
$$

where $F_{a}>0$ is the frequency factor, $E_{a}$ is the activation energy, $T$ is the temperature (in $\mathrm{K}$ ), and $R$ is the universal gas constant $\left(\approx 8.31 \mathrm{~J} \mathrm{~K}^{-1} \mathrm{~mol}^{-1}\right)$. The values $F_{a}$ and $E_{a}$ are fixed for each reaction (e.g., for water formation, $\mathrm{OH}+\mathrm{H}_{2} \stackrel{k}{\rightarrow} \mathrm{H}_{2} \mathrm{O}+\mathrm{H}$, $F_{a}=8 \times 10^{10} \mathrm{~L} \mathrm{~mol}^{-1} \mathrm{~s}^{-1}$, and $\left.E_{a}=42 \times 10^{3} \mathrm{~J} \mathrm{~mol}^{-1}\right)$. For most reactions $E_{a}>0$, so that $k$ increases with the temperature. Then we have (note that $4 / c$ is a Lipschitz constant for the function $e^{-c / x}$ )

$$
\left|k\left(T_{1}\right)-k\left(T_{0}\right)\right|=F_{a}\left|e^{-\frac{E_{a}}{R T_{1}}}-e^{-\frac{E_{a}}{R T_{0}}}\right| \leq 4 R \frac{F_{a}}{E_{a}}\left|T_{1}-T_{0}\right| .
$$

In general, changes in temperature will be reflected in the matrix of kinetic parameters as $\left\|u^{T_{1}}-u^{T_{0}}\right\| \leq c\left|T_{1}-T_{0}\right|$ for some $c>0$. Then, from Theorem 3.6, we expect that a change in temperature from $T_{0}$ to $T_{1}$ will lead to a deviation in the steady state of order $\left|\bar{x}_{1}-\bar{x}_{0}\right| \lesssim \varphi\left(\left|T_{1}-T_{0}\right|\right)$, where $\varphi$ is some $\mathcal{K}_{\infty}$ function.

Example. Consider the model in Figure 1 and assume that the concentration of ligand is regulated from "outside." For instance, $L(t)$ may be experimentally designed to be a piecewise constant function, in order to measure the response of the system to different concentrations of ligand. Or $L$ could be regulated by an independent network. In either case, we would have the following system:

$$
\dot{x}=f(x, w),
$$


where $x=\left(R_{1}, R_{2}, C_{1}, C_{2}\right)^{\prime}$ and

$$
w(t)=\left(\begin{array}{cccc}
0 & a_{12} & a_{13} L(t) & 0 \\
a_{21} L(t) & 0 & 0 & a_{24} \\
a_{31} L(t) & 0 & 0 & a_{34} \\
0 & a_{42} & a_{43} L(t) & 0
\end{array}\right) .
$$

If $L$ is determined by a dynamical system, say $\dot{z}=g(z)$, also of the form (2.1), then we know that $z(t) \rightarrow \bar{z}$ for some $\bar{z} \in \mathcal{E}$, and therefore $w(\cdot) \in \mathcal{W}$. An interesting problem for further analysis is whether the convergence of $L(t)$ to some $\bar{L}$ implies that the trajectory $x(t)$ will also converge to some $\bar{x} \in \mathcal{E}$. Another interesting question, which we leave for further research, is whether the cascade system $\dot{x}=f(x, z), \dot{z}=g(z)$ is again input-to-state stable, in the manner developed in [15].

4. Dependence of the steady states on the kinetic parameters. A typical problem concerning cell receptor-ligand interactions, and many other biochemical reactions, is to determine the "dose-response" curves, that is, determine the final concentration of the products, $\bar{C}_{1}$ or $\bar{C}_{2}$, as a function of the initial concentration of ligand, $L_{0}$ (see $[12,19]$ and [5]). When translated into mathematical language, this problem involves the characterization of the multiple steady states of system (2.4) and their dependence on the matrix $A$ and classes $\mathcal{S}_{x_{0}}$.

We recall that, for the case of constant inputs, say $u(t) \equiv A$, the system $\dot{x}=$ $f(x, u)$ with initial condition $x(0)=x_{0}$ converges to the constant steady state $\bar{x}\left(x_{0}, A\right)$. In contrast, for a general input $u(\cdot)$ one certainly does not expect the system to converge to a constant steady state. However, one may still consider the map $\bar{x}$ : $\mathbb{R}_{>0}^{n} \times \mathcal{A}_{\geq 0} \rightarrow \mathcal{E}$, where $\bar{x}\left(x_{0}, A\right)$ is defined as the unique positive steady state of the system $\dot{x}=f(x, A)=f_{A}(x)$ in the class $\mathcal{S}_{x_{0}}$. Then the following is true:

$$
\bar{x}\left(x_{0}, u(t)\right) \in \mathcal{E} \quad \forall t .
$$

We will show that $\bar{x}$ is in fact a real analytic function of $x_{0}$ and $A$. This will help us in the proof of the main results, namely, in section 5 , to show that the system is $\mathbb{R}_{\geq 0}^{n}$-forward complete.

ThEOREM 4.1. Assume that the maps $\theta_{i}$ are real analytic functions. Then the map $\bar{x}: \mathbb{R}_{>0}^{n} \times \mathcal{A}_{\geq 0} \rightarrow \mathcal{E} \subset \mathbb{R}_{>0}^{n}$ given by $\left(x_{0}, A\right) \mapsto \bar{x}\left(x_{0}, A\right)$ is real analytic.

To prove this theorem we will use the following alternative expression for $f_{A}$ (see [17]):

$$
f_{A}(x)=B \tilde{A} \theta_{B}(x),
$$

where

$$
\theta_{B}(x)=\left(\begin{array}{c}
\theta_{1}\left(x_{1}\right)^{b_{11}} \theta_{2}\left(x_{2}\right)^{b_{21}} \cdots \theta_{n}\left(x_{n}\right)^{b_{n 1}} \\
\theta_{1}\left(x_{1}\right)^{b_{12}} \theta_{2}\left(x_{2}\right)^{b_{22}} \cdots \theta_{n}\left(x_{n}\right)^{b_{n 2}} \\
\vdots \\
\theta_{1}\left(x_{1}\right)^{b_{1 m}} \theta_{2}\left(x_{2}\right)^{b_{2 m}} \cdots \theta_{n}\left(x_{n}\right)^{b_{n m}}
\end{array}\right)=\exp \left[B^{\prime} \rho(x)\right]
$$

and

$$
\tilde{A}=A+\left(\begin{array}{cccc}
-\sum_{i=1}^{m} a_{i 1} & 0 & \cdots & 0 \\
0 & -\sum_{i=1}^{m} a_{i 2} & \cdots & 0 \\
\vdots & \vdots & & \vdots \\
0 & 0 & \cdots & -\sum_{i=1}^{m} a_{i m}
\end{array}\right)
$$


(Recall that we assumed without loss of generality that all the diagonal entries of $A$ are zero.) Now, given any matrix $G \in \mathbb{R}^{m \times m}$, with entries $g_{i j}$, define

$$
\phi(G)=\left(1+\sum_{i=1}^{m} g_{i i}^{2}\right)^{-1} \quad \text { and } \quad M_{G}=(\phi(G) G+I)^{m-1} .
$$

By construction, the diagonal entries of $\phi(G) G+I$ are positive. Introduce the following subset of $\mathbb{R}^{m \times m}$ :

$$
\mathcal{G}=\left\{G \in \mathbb{R}^{m \times m}: M_{G}>0 \text { and } \overline{1} G=0\right\},
$$

where the inequality means that every entry of the matrix on the left-hand side is strictly positive, and $\overline{1}$ is the row vector $(11 \cdots 1)$. The set $\mathcal{G}$ may be seen as an open subset of the $\left(m^{2}-m\right)$-dimensional linear subspace $\{G: \overline{1} G=0\}$ of $\mathbb{R}^{m \times m}$. Define $\mathcal{G}_{\geq 0}$ to be the set of all irreducible matrices which have $\overline{1} G=0$, nonnegative off-diagonal entries and arbitrary diagonal entries. Note that

$$
\mathcal{G}_{\geq 0}=\{G \in \mathcal{G}: G \text { has nonnegative off-diagonal entries }\} .
$$

Then to each matrix $A \in \mathcal{A}_{\geq 0}$, we associate a matrix $\tilde{A} \in \mathcal{G}_{\geq 0}$ : clearly, $\overline{1} \tilde{A}=0$ and so $\tilde{A} \in \mathcal{G}_{\geq 0}$.

For each $G \in \mathcal{G}$ observe that $\overline{1} M_{G}=\overline{1}(\phi(G) G+I)^{m-1}=\overline{1}$ because $\overline{1} G=0$ and $\overline{1}(\phi(G) G+I)=\overline{1}$. So, any nonnegative eigenvector, $v \in \mathbb{R}_{\geq 0}^{n}$, of the matrix $M_{G}$ must correspond to the eigenvalue $\mu=1$ since

$$
M_{G} v=\mu v \Rightarrow \overline{1}\left(M_{G} v\right)=\overline{1}(\mu v) \Leftrightarrow \overline{1} v=\mu \overline{1} v
$$

and $\overline{1} v$ is a positive scalar (since $v \neq(0, \ldots, 0)^{\prime}$ by the definition of eigenvector).

Since, by definition, $M_{G}$ is irreducible and has all entries positive, by the PerronFrobenius theorem we know that the spectral radius of $M_{G}, \sigma\left(M_{G}\right)$, is an eigenvalue of $M_{G}$ of algebraic (and hence geometric) multiplicity one. Moreover, an eigenvector associated with $\sigma\left(M_{G}\right)$ can be chosen to have all entries strictly positive (this will be a Perron eigenvector of $M_{G}$, and any two such vectors are positive multiples of each other). But, as we have just seen, any positive eigenvector of $M_{G}$ corresponds to the eigenvalue $\mu=1$, so we have

$$
\sigma\left(M_{G}\right) \equiv 1 \quad \forall G \in \mathcal{G} .
$$

Define $v_{\mathrm{P}}: \mathcal{G} \rightarrow \mathbb{R}_{>0}^{m}$ to be the map that assigns to each $G \in \mathcal{G}$ the unique Perron eigenvector of $M_{G}$, which has its first coordinate equal to 1 ,

$$
v_{\mathrm{P}}=\left(\begin{array}{c}
1 \\
w_{\mathrm{P}}
\end{array}\right)
$$

for some $w_{\mathrm{P}} \in \mathbb{R}_{>0}^{m-1}$. Then the map $v_{\mathrm{P}}$ is a rational function on $\mathcal{G}$, as shown in the appendix.

Proof of Theorem 4.1. A function $f$, defined on an open set $\mathcal{V}$, is real analytic if it admits a power series expansion on a neighborhood of each point of $\mathcal{V}$. If, as in our case, the set $\mathcal{V}$ is not open, then the function $f$ is still called real analytic if it admits an extension to a real analytic function on a neighborhood of $\mathcal{V}$ (see [16]). This is what we will show for the map $\bar{x}(\cdot, \cdot)$. 
For each $A$ consider the matrix $\tilde{A} \in \mathcal{G}_{\geq 0}$, constructed from $A$ as indicated above. Then, from (A.1), $\operatorname{ker} \tilde{A}=\operatorname{span}\left\{v_{\mathrm{P}}(\tilde{A})\right\}$. Because $B$ has full column rank, it follows that each equilibrium $\bar{x} \in E_{A,+}$ is characterized by

$$
\theta_{B}(\bar{x})=c v_{\mathrm{P}}(\tilde{A}) \Leftrightarrow B^{\prime} \rho(\bar{x})=\rho\left(c v_{\mathrm{P}}(\tilde{A})\right),
$$

where $c$ is a positive constant.

Claim. For each $A$, the element $\bar{z}(A) \in \mathbb{R}_{>0}^{n}$ given by

$$
\bar{z}(A)=\exp \left[B\left(B^{\prime} B\right)^{-1} \rho\left(v_{\mathrm{P}}(\tilde{A})\right)\right]
$$

is an equilibrium point in $E_{A,+}$.

To prove the claim, note that $B$ has full column rank, so $B^{\prime} B$ is an invertible matrix and the formula gives $\rho(\bar{z}(A))=B\left(B^{\prime} B\right)^{-1} \rho\left(v_{\mathrm{P}}(\tilde{A})\right)$ or, equivalently,

$$
B^{\prime} \rho(\bar{z}(A))=B^{\prime} B\left(B^{\prime} B\right)^{-1} \rho\left(v_{\mathrm{P}}(\tilde{A})\right)=\rho\left(v_{\mathrm{P}}(\tilde{A})\right) .
$$

The claim is proved by letting $c=1$ and $\bar{x}=\bar{z}(A)$ in (4.2).

Now, by Proposition A.1, the map $v_{\mathrm{P}}$ is a rational function on $\mathcal{G}$ and, furthermore, $v_{\mathrm{P}}(G) \in \mathbb{R}_{>0}^{m}$. The functions $\exp (\cdot)$ and $\rho(\cdot)$ are analytic on $\mathbb{R}^{n}$ and $\mathbb{R}_{>0}^{n}$, respectively, so it follows that the map $\tilde{A} \mapsto A(\tilde{A}) \mapsto \bar{z}(A)$ from $\mathcal{G}_{\geq 0} \rightarrow \mathcal{E}$ is analytic because it admits an analytic extension to $\mathcal{G} \rightarrow \mathbb{R}_{>0}^{n}$. (Denote by $A(\tilde{A})$ the matrix which coincides with $A$ on the off-diagonal entries and has zero in its diagonal.)

Next, from Lemma IV.1 (and proof of Theorem 2) in [17], there is a real analytic map $\varphi(q, w)$, defined on $\mathbb{R}_{>0}^{n} \times \mathbb{R}_{>0}^{n}$, such that, for each $q \in \mathbb{R}_{>0}^{n}, x=\varphi(q, \bar{z}(A))$ is the unique positive equilibrium of the system $\dot{x}=f_{A}(x)$ in the same class of $q$. Let $q=x_{0}$ and $w=\bar{z}(A)$. We may now conclude that the map $\mathbb{R}_{>0}^{n} \times \mathcal{G}_{\geq 0} \rightarrow \mathcal{E}$ given by

$$
\left(x_{0}, \tilde{A}\right) \mapsto \varphi\left(x_{0}, \bar{z}(A)\right)
$$

is again analytic because it admits an analytic extension to $\mathbb{R}_{>0}^{n} \times \mathcal{G}$. Therefore,

$$
\bar{x}\left(x_{0}, A\right) \equiv \varphi\left(x_{0}, \bar{z}(A)\right)
$$

is the unique element that belongs to both the class of $x_{0}$ and the equilibria set $E_{A,+}$, and we have just shown that the map $\bar{x}: \mathbb{R}_{>0}^{n} \times \mathcal{A}_{\geq 0} \rightarrow \mathcal{E}$ is real analytic.

5. Existence and completeness of solutions. We now turn our attention to system (2.3) and will show that it is complete in the sense of Definition 3.1.

Proposition 5.1. Consider system (2.3), with state space $\mathcal{X}=\mathbb{R}^{n}$ and inputvalue set $\mathcal{A}_{\geq 0}$. Then the system is $\mathbb{R}_{>0}^{n}$-forward invariant.

Proof. Given an initial condition $x(0)=x_{0} \in \mathbb{R}_{>0}^{n}$ and an $\mathcal{A}_{\geq 0}$-valued input $u(t)$, define $F(t, x):=f(x, u(t))$. The existence and uniqueness of a maximal solution to this initial-value problem follows from standard results (such as stated in [16]), by noticing that, for each fixed $t, F(t, x)$ is locally Lipschitz in $x$ and, for each fixed $x$, it is locally integrable as a function of time. Forward invariance also follows from standard arguments based on the fact that, for $x \in \mathbb{R}_{>0}^{n}$, if $x_{k}=0$ for any $k$, then $F_{k}(t, x) \geq 0$. The actual proof is very similar to that of Proposition 3.13 in [4], so we will not reproduce it here. In that proposition, simply take $C=0$ and replace " $a_{i j}$ " by " $u_{i j}$ " (we only use the fact that $u_{i j} \geq 0$ ). 
5.1. A Lyapunov function. In order to prove $\mathbb{R}_{>0}^{n}$-forward completeness of system (2.3), we will need to introduce our candidate ISS-Lyapunov function. Fix any point $\bar{x}$ in $\mathcal{E}$ and recall the notation $\rho_{i}=\ln \theta_{i}$. Define

$$
V(x, \bar{x})=\sum_{i=1}^{n} \int_{\bar{x}_{i}}^{x_{i}}\left(\rho_{i}(s)-\rho_{i}\left(\bar{x}_{i}\right)\right) d s .
$$

This function is introduced and motivated in [17], where it is shown that $V$ is always nonnegative and zero if and only if $x \equiv \bar{x}$. It is also easy to see that $V(x, \bar{x}) \rightarrow+\infty$ as $|x-\bar{x}| \rightarrow+\infty$. Also, the function $V$ is proper in the following sense: for each compact set $P \subset \mathcal{E}$, one can show that there exist two class $\mathcal{K}_{\infty}$ functions $\nu_{1}=\nu_{1, P}$, $\nu_{2}=\nu_{2, P}$ such that

$$
\nu_{1}(|x-\bar{x}|) \leq V(x, \bar{x}) \leq \nu_{2}(|x-\bar{x}|)
$$

$\forall x \in \mathbb{R}_{\geq 0}^{n}$ and $\forall \bar{x} \in P$. For instance, we may take

$$
\nu_{1}(r)=\inf \left\{V(x, \bar{x}):|x-\bar{x}| \geq r, x \in \mathbb{R}_{\geq 0}^{n}, \bar{x} \in P\right\}
$$

and

$$
\nu_{2}(r)=r+\max \left\{V(x, \bar{x}):|x-\bar{x}| \leq r, x \in \mathbb{R}_{\geq 0}^{n}, \bar{x} \in P\right\} .
$$

So, it is easy to see that $V$ satisfies both properties (i) and (ii) of Definition 3.3. In the case of maps $\theta_{i}(r)=|r|$, the function has the form

$$
V(x, \bar{x})=\sum_{i=1}^{n} x_{i}\left(\ln x_{i}-\ln \bar{x}_{i}\right)+\left(\bar{x}_{i}-x_{i}\right) .
$$

Some more notation will be useful. For any $\bar{x}=\left(\bar{x}_{1}, \ldots, \bar{x}_{n}\right)^{\prime} \in \mathcal{E}$ and $\forall x \in \mathbb{R}_{>0}^{n}$ define

$$
q_{j}(x, \bar{x})=q_{j}:=\left\langle b_{j}, \rho(x)-\rho(\bar{x})\right\rangle .
$$

Introduce also the scalar function $\omega: \mathbb{R} \rightarrow \mathbb{R}_{\geq 0}$ given by

$$
\omega(r)=e^{r}-1-r .
$$

Furthermore, note that

$$
\begin{aligned}
& \nabla_{x} V(x, \bar{x})=\rho(x)-\rho(\bar{x})=\left(\ln \theta_{1}\left(x_{1}\right)-\ln \theta_{1}\left(\bar{x}_{1}\right), \ldots, \ln \theta_{n}\left(x_{n}\right)-\ln \theta_{n}\left(\bar{x}_{n}\right)\right), \\
& \nabla_{\bar{x}} V(x, \bar{x})=\left(\left(\bar{x}_{1}-x_{1}\right) \frac{\theta_{1}^{\prime}\left(\bar{x}_{1}\right)}{\theta_{1}\left(\bar{x}_{1}\right)}, \ldots,\left(\bar{x}_{n}-x_{n}\right) \frac{\theta_{n}^{\prime}\left(\bar{x}_{n}\right)}{\theta_{n}\left(\bar{x}_{n}\right)}\right) .
\end{aligned}
$$

Now, given any $A \in \mathcal{A}_{\geq 0}$ and any $\bar{x} \in E_{A,+}$, consider

$$
\begin{aligned}
\nabla V(x, \bar{x}) f_{A}(x) & =\left\langle\rho(x)-\rho(\bar{x}), f_{A}(x)\right\rangle \\
& =\sum_{i=1}^{m} \sum_{j=1}^{m} a_{i j} e^{\left\langle b_{j}, \rho(\bar{x})\right\rangle} e^{q_{j}}\left(q_{i}-q_{j}\right) \\
& =-\sum_{i=1}^{m} \sum_{j=1}^{m} a_{i j} e^{\left\langle b_{j}, \rho(\bar{x})\right\rangle} e^{q_{j}} \omega\left(q_{i}-q_{j}\right) \\
& =:-W(x, \bar{x}) .
\end{aligned}
$$


The third inequality holds because

$$
\begin{aligned}
e^{q_{j}}\left(q_{i}-q_{j}\right) & =e^{q_{j}}\left(q_{i}-q_{j}\right)-e^{q_{j}}\left(e^{q_{i}-q_{j}}-1\right)+e^{q_{j}}\left(e^{q_{i}-q_{j}}-1\right) \\
& =-e^{q_{j}} \omega\left(q_{i}-q_{j}\right)+\left(e^{q_{i}}-e^{q_{j}}\right)
\end{aligned}
$$

and

$$
\begin{aligned}
& \sum_{i=1}^{m} \sum_{j=1}^{m} a_{i j} e^{\left\langle b_{j}, \rho(\bar{x})\right\rangle}\left(e^{q_{i}}-e^{q_{j}}\right) \\
= & \left(e^{q_{1}}, \ldots, e^{q_{m}}\right)^{\prime} A \theta_{B}(\bar{x})-\left(e^{q_{1}}, \ldots, e^{q_{m}}\right)^{\prime} \operatorname{diag}\left(\sum_{i} a_{i 1}, \ldots, \sum_{i} a_{i m}\right) \theta_{B}(\bar{x}) \\
= & \left(e^{q_{1}}, \ldots, e^{q_{m}}\right)^{\prime} \tilde{A} \theta_{B}(\bar{x})=0,
\end{aligned}
$$

since at steady state, recalling (4.1) and that $B$ has full rank,

$$
f(\bar{x}, A)=f_{A}(\bar{x})=B \tilde{A} \theta_{B}(\bar{x})=0 .
$$

An important point to notice is that $-W(x, \bar{x})$ (hence $\left.\nabla V(x, \bar{x}) f_{A}(x)\right)$ is always nonpositive, because $\omega(r) \geq 0 \forall r$ (with $\omega(r)=0$ if and only if $r=0$ ).

To prove $\mathbb{R}_{>0}^{n}$-forward completeness, we consider the function $V\left(x(t), \bar{x}\left(x_{0}, u(t)\right)\right)$ along a trajectory $x\left(\cdot, x_{0}, u(\cdot)\right)$, which is the solution of $(2.3)$, when the input is $u(\cdot)$ and the initial condition $x(0)=x_{0}$. For the next lemma recall that the maps $\theta_{i}$ are onto an interval of the form $\left[0, \sigma_{i}\right)$, where $0<\sigma_{i} \leq+\infty$.

Lemma 5.2. Given any compact set $P \subset \mathcal{E}$, let $\ell_{1}, \ell_{2}>0$ be any numbers so that

$$
e^{\frac{1}{\ell_{1}}}<\frac{\sigma_{i}}{\theta_{i}\left(\bar{x}_{i}\right)} \quad \forall \bar{x} \in P, \quad \forall i=1, \ldots, n
$$

and

$$
\ell_{2} \bar{x}_{i}>\left|\bar{x}_{i}-r_{ \pm}\right| \quad \forall \bar{x} \in P, \quad \forall i=1, \ldots, n,
$$

where the numbers $r_{ \pm}$are defined by the equations $\ln \theta_{i}\left(r_{ \pm}\right)=\ln \theta_{i}\left(\bar{x}_{i}\right) \pm 1 / \ell_{1}$.

Then

$$
\left|\bar{x}_{i}-x_{i}\right| \leq \ell_{1} V(x, \bar{x})+\ell_{2} \bar{x}_{i}
$$

$\forall x \in \mathbb{R}_{\geq 0}^{n}$ and $\forall \bar{x} \in P$.

Remark. If $\theta_{i}(r)=|r| \forall i=1, \ldots, n$, then $r_{ \pm}=e^{ \pm \frac{1}{\ell_{1}}} \bar{x}_{i}$, and we may choose $\ell_{1}$ and $\ell_{2}$ independently of $P$ : indeed, condition (5.8) becomes $e^{\frac{1}{\ell_{1}}}<\infty$ (satisfied by any $\ell_{1}>0$ ), and condition (5.9) becomes $\ell_{2}>\left|1-e^{ \pm \frac{1}{\ell_{1}}}\right|$. For instance, we may pick $\ell_{1}=1$ and $\ell_{2}=2$.

Proof. Pick any compact set $P \subset \mathcal{E}$ and pick any numbers $\ell_{1}$ and $\ell_{2}$ according to (5.8) and (5.9). First, note that the definition of $V$ implies (see (5.1))

$$
\int_{\bar{x}_{i}}^{x_{i}} \rho_{i}(s)-\rho_{i}\left(\bar{x}_{i}\right) d s \leq V(x, \bar{x}), \quad i=1, \ldots, n
$$

(recall that $\left.\rho_{i}(s)=\ln \theta_{i}(s)\right)$. Now fix any $i \in\{1, \ldots, n\}$ and put $a=\bar{x}_{i}$. For $r \geq 0$, $a>0$, define

$$
h(r)=\ell_{1} \int_{a}^{r} \rho_{i}(s)-\rho_{i}(a) d s-|a-r|+\ell_{2} a .
$$


We will show that $h(r) \geq 0 \forall r \geq 0$. The first derivative of $h$ is piecewise continuous

$$
\frac{d h}{d r}= \begin{cases}\ell_{1}\left(\rho_{i}(r)-\rho_{i}(a)\right)+1 & \text { if } 0 \leq r<a, \\ \ell_{1}\left(\rho_{i}(r)-\rho_{i}(a)\right)-1 & \text { if } r>a\end{cases}
$$

and the second derivative is

$$
\frac{d^{2} h}{d r^{2}}=\ell_{1} \frac{\theta_{i}^{\prime}(r)}{\theta_{i}(r)}>0 \quad \text { for } \quad r \neq a,
$$

where $\theta_{i}^{\prime}(r)=d \theta_{i} / d r>0$, because $\theta_{i}$ is strictly increasing. Each continuous piece of the derivative has a zero, at the points $r_{ \pm}$,

$$
\frac{d h}{d r}=0 \Leftrightarrow \begin{cases}\rho_{i}\left(r_{-}\right)=-\frac{1}{\ell_{1}}+\rho_{i}(a) & \text { if } 0<r<a, \\ \rho_{i}\left(r_{+}\right)=\frac{1}{\ell_{1}}+\rho_{i}(a) & \text { if } r>a .\end{cases}
$$

Note that, because $\rho_{i}$ is an increasing function, indeed $r_{-}<a$ and $r_{+}>a$. In addition, from (5.8) it follows that both $r_{-}$and $r_{+}$are well defined, since they belong to the domain of $\theta_{i}$. Since the second derivative of $h$ is always positive for $r \neq a, h$ has local minima at the points $r=r_{ \pm}$. By definition of $\ell_{2}$, it follows that the value of $h$ at $r_{ \pm}$is positive:

$$
h\left(r_{ \pm}\right)=\ell_{1} \int_{a}^{r_{ \pm}} \rho_{i}(s)-\rho_{i}(a) d s-\left|a-r_{ \pm}\right|+\ell_{2} a,
$$

since the first term is positive by construction of $V$ and the two other terms satisfy (5.9)

$$
-\left|a-r_{ \pm}\right|+\ell_{2} a>0
$$

To summarize,

$$
\frac{d h}{d r}= \begin{cases}<0, & 0 \leq r<r_{-}, \\ >0, & r_{-}<r<a, \\ <0, & a<r<r_{+}, \\ >0, & r_{+}<r\end{cases}
$$

so that $h$ decreases down to a local positive minimum at $r_{-}$, then increases up to $h(a)>0$, and decreases again to another local positive minimum at $r_{+}$, and increases $\forall r>r_{+}$. Therefore,

$$
h(r)>0 \quad \forall r \in[0,+\infty) .
$$

This finishes the proof, since for each $a=\bar{x}_{i}$,

$$
\begin{aligned}
h(r)>0 \Leftrightarrow\left|\bar{x}_{i}-x_{i}\right| & \leq \ell_{1} \int_{\bar{x}_{i}}^{x_{i}} \rho_{i}(s)-\rho_{i}\left(\bar{x}_{i}\right) d s+\ell_{2} \bar{x}_{i} \\
& \leq \ell_{1} V\left(x, \bar{x}_{i}\right)+\ell_{2} \bar{x}_{i} .
\end{aligned}
$$

\subsection{Completeness.}

Proposition 5.3. Consider system (2.3) with state space $\mathcal{X}=\mathbb{R}^{n}$. Then the system is $\mathbb{R}_{>0}^{n}$-forward complete, whenever the input map $u(\cdot)$ is in $\mathcal{W}$.

Proof. Pick any input map $u(\cdot)$ in $\mathcal{W}$. Let $x(t)$ be the issuing solution of (2.3), with the initial condition $x(0)=x_{0} \in \mathbb{R}_{>0}^{n}$, and let it be defined on the maximal interval 
$[0, T)$. From Proposition 5.1, we already know that $x(t):=x\left(t, x_{0}, u(\cdot)\right) \in \mathbb{R}_{>0}^{n} \forall t$ in the interval $[0, T)$. Assuming that $T<+\infty$, we will show that $x(\cdot)$ evolves on a compact subset of $\mathcal{X} \forall t \in[0, T)$, which is a contradiction. To do this, we consider the function

$$
g(t)=V\left(x(t), \bar{x}\left(x_{0}, u(t)\right)\right)
$$

whose derivative is

$$
\begin{aligned}
\dot{g}(t) & =\nabla_{x} V\left(x(t), \bar{x}\left(x_{0}, u(t)\right)\right) \frac{d}{d t}[x(t)]+\nabla_{\bar{x}} V\left(x(t), \bar{x}\left(x_{0}, u(t)\right)\right) \frac{d}{d t}\left[\bar{x}\left(x_{0}, u(t)\right)\right] \\
& =\langle\rho(x)-\rho(\bar{x}), f(x, u)\rangle+\sum_{i=1}^{n}\left(\bar{x}_{i}-x_{i}\right) \dot{\bar{x}}_{i} \frac{\theta_{i}^{\prime}\left(\bar{x}_{i}\right)}{\theta_{i}\left(\bar{x}_{i}\right)}
\end{aligned}
$$

where, for simplicity, we used $x \equiv x(t)$ and $\bar{x} \equiv \bar{x}\left(x_{0}, u(t)\right)$, and $\dot{\bar{x}}_{i}:=\frac{d}{d t}\left[\bar{x}_{i}\left(x_{0}, u(t)\right)\right]$.

Now, for almost all $t \in[0, T), u(t)$ takes values in a compact set. So there exist constants $\underline{c}, \bar{c}>0$ such that

$$
\underline{c} \leq\left|\bar{x}_{i}\left(x_{0}, u(t)\right)\right| \leq \bar{c} \text { for almost all } t \in[0, T) .
$$

By differentiability of $\bar{x}(\cdot, \cdot)$ (Theorem 4.1), and because $u$ is piecewise locally Lipschitz with finitely many points of discontinuity, there exist positive constants $\kappa$ and $c_{1}$ such that

$$
\dot{\bar{x}}_{i}=\sum_{i, j=1}^{m} \frac{d \bar{x}_{i}}{d u_{i j}} \frac{d u_{i j}}{d t} \leq \sum_{i, j=1}^{m} \kappa\left|\frac{d \bar{x}_{i}}{d u_{i j}}\right| \leq c_{1} \quad \text { for almost all } t \in[0, T) .
$$

The function $\theta_{i}$ is positive and strictly increasing, so $\theta_{i}^{\prime}(r)$ is also positive. Since $\bar{x}_{i}(\cdot, \cdot)$ takes values in a compact set, there exists $c_{2}>0$ such that

$$
\frac{\theta_{i}^{\prime}\left(\bar{x}_{i}\right)}{\theta_{i}\left(\bar{x}_{i}\right)} \leq c_{2} \quad \text { for almost all } t \in[0, T)
$$

From (5.6), $\left\langle\rho(x)-\rho\left(\bar{x}\left(x_{0}, u\right)\right), f(x, u)\right\rangle \leq 0 \forall x \in \mathbb{R}_{>0}^{n}, u \in \mathcal{A}_{\geq 0}$. Then, applying Lemma 5.2, with $P=[\underline{c}, \bar{c}]^{n} \cap \mathcal{E}$, to the second term on $\dot{g}$, we obtain

$$
\dot{g} \leq \sum_{i=1}^{n}\left|\dot{\bar{x}}_{i}\right| \frac{\theta_{i}^{\prime}\left(\bar{x}_{i}\right)}{\theta_{i}\left(\bar{x}_{i}\right)}\left(\ell_{1} V(x, \bar{x})+\ell_{2} \bar{x}_{i}\right)
$$

which implies

$$
\dot{g}(t) \leq \ell_{1} c_{1} c_{2} g(t)+\ell_{2} c_{1} c_{2} \bar{c} \quad \text { for almost all } t \in[0, T) .
$$

Taking $c_{3}=\ell_{2} c_{1} c_{2} \bar{c}$ and $c_{4}=\ell_{1} c_{1} c_{2}$ and applying Gronwall's lemma, yield

$$
g(t) \leq c_{3} e^{c_{4} T} \quad \forall t \in[0, T)
$$

For the compact set $P=[\underline{c}, \bar{c}]^{n} \cap \mathcal{E}$, let $\nu_{1}=\nu_{1, P}$ be the class $\mathcal{K}_{\infty}$ function such that $\nu_{1}(|x-\bar{x}|) \leq V(x, \bar{x}) \forall x \in \mathbb{R}_{\geq 0}^{n}$ and $\bar{x} \in P$. Thus,

$$
\nu_{1}\left(\left|x(t)-\bar{x}\left(x_{0}, u(t)\right)\right|\right) \leq g(t) \quad \forall t \in[0, T)
$$

and, therefore,

$$
|x(t)| \leq \bar{c}+\nu_{1}^{-1}\left(c_{3} e^{c_{4} T}\right)
$$

implying that $x$ evolves in a compact subset of the state space, which contradicts $T<+\infty$. 
6. ISS estimates. To establish the main results, we now show that the function $V$ is a uniformly semiglobal ISS-Lyapunov function. In section 5.1 it was shown that $V$ satisfies properties (i) and (ii) of Definition 3.3. We next show that it also satisfies property (iii).

For any $\bar{x}=\left(\bar{x}_{1}, \ldots, \bar{x}_{n}\right)^{\prime} \in \mathcal{E}$ and any $x \in \mathbb{R}_{\geq 0}^{n}$ define

$$
\pi_{j}(x, \bar{x})=\pi_{j}:=\left[\frac{\theta_{1}\left(x_{1}\right)}{\theta_{1}\left(\bar{x}_{1}\right)}\right]^{b_{1 j}}\left[\frac{\theta_{2}\left(x_{2}\right)}{\theta_{2}\left(\bar{x}_{2}\right)}\right]^{b_{2 j}} \cdots\left[\frac{\theta_{n}\left(x_{n}\right)}{\theta_{n}\left(\bar{x}_{n}\right)}\right]^{b_{n j}}
$$

and observe that, if $x \in \mathbb{R}_{>0}^{n}$, from (5.4)

$$
\pi_{j}=e^{q_{j}} .
$$

Using this notation, define the function $\Psi: \mathbb{R}_{\geq 0}^{n} \times \mathcal{E} \rightarrow \mathbb{R}_{\geq 0}$,

$$
\Psi(x, \bar{x}):=\sum_{i=1}^{m} \sum_{j=1}^{m}\left(e^{-\pi_{i}}-e^{-\pi_{j}}\right)^{2},
$$

which, from Lemma 3.8 in [4], satisfies

$$
\Psi(x, \bar{x})=0 \quad \Leftrightarrow \quad x \in E_{0} \cup E_{A,+},
$$

where $E_{0}$ is the set of boundary equilibria and $A \in \mathcal{A}_{\geq 0}$ is such that $\bar{x} \in E_{A,+}$. Recall the function $W$ defined in (5.6): a useful estimate (see Lemma 3.10 in [4]) establishes that, for each fixed $\bar{x}$,

$$
W(x, \bar{x}) \geq \frac{1}{2} \sum_{i=1}^{m} \sum_{j=1}^{m} a_{i j} e^{\left\langle b_{j}, \rho(\bar{x})\right\rangle}\left(e^{-\pi_{i}}-e^{-\pi_{j}}\right)^{2} \quad \forall x \in \mathbb{R}_{>0}^{n} .
$$

Moreover, since $x \in \mathbb{R}_{>0}^{n}$, it follows from (6.2) that the expression on the right-hand side is zero if and only if $x \equiv \bar{x}$.

Next, given any $A \in \mathcal{A}_{\geq 0}$, suppose that $A_{1}$ is a matrix with entries $a_{i j}^{1}=1$ if $a_{i j}>0$ and $a_{i j}^{1}=0$ if $a_{i j}=0$. Then, for expression (6.3), we can write

$$
W(x, \bar{x}) \geq \frac{1}{2} \min _{a_{i j}>0}\left\{a_{i j}\right\} \min _{j} e^{\left\langle b_{j}, \rho(\bar{x})\right\rangle} \sum_{i=1}^{m} \sum_{j=1}^{m} a_{i j}^{1}\left(e^{-\pi_{i}}-e^{-\pi_{j}}\right)^{2} .
$$

Since $A_{1}$ is irreducible, we may apply Lemma VIII.1 from [17] to conclude that there exists a positive constant $\kappa\left(A_{1}\right)$ such that

$$
\sum_{i=1}^{m} \sum_{j=1}^{m} a_{i j}^{1}\left(e^{-\pi_{i}}-e^{-\pi_{j}}\right)^{2} \geq \kappa\left(A_{1}\right) \sum_{i=1}^{m} \sum_{j=1}^{m}\left(e^{-\pi_{i}}-e^{-\pi_{j}}\right)^{2} .
$$

Now, define the following subset of $\mathcal{A}_{\geq 0}$,

$$
\mathcal{A}_{\geq 0}^{1}:=\left\{A \in \mathcal{A}_{\geq 0}: a_{i j}=1 \text { or } a_{i j}=0\right\},
$$

and note that its cardinality is finite (in fact, the number of elements in $\mathcal{A}_{\geq 0}^{1}$ is equal to the number of distinct strongly connected graphs with $m$ vertexes). Then let

$$
\kappa_{1}:=\min \left\{\kappa(A): A \in \mathcal{A}_{\geq 0}^{1}\right\} .
$$


Additionally observe that, given any $\varepsilon>0$,

$$
A \in \mathbb{U}_{\varepsilon} \quad \text { satisfies } \quad \min _{a_{i j}>0}\left\{a_{i j}\right\}=\varepsilon
$$

(where $\mathbb{U}_{\varepsilon}$ is the set defined in (3.2)).

From this discussion, it is easy to establish the following lemmas.

Lemma 6.1. For each compact $P \subset \mathcal{E}$ and each $\varepsilon>0$, there exists a constant $c(P, \varepsilon)$ given by

$$
c(P, \varepsilon)=\frac{1}{2} \varepsilon \kappa_{1} \min _{\bar{x} \in P} \min _{j} e^{\left\langle b_{j}, \rho(\bar{x})\right\rangle}
$$

such that

$$
W(x, \bar{x}) \geq c(P, \varepsilon) \Psi(x, \bar{x})
$$

$\forall x \in \mathbb{R}_{>0}^{n}$ and any element $\bar{x} \in P$.

Lemma 6.2. Let $P \subset \mathcal{E}$ be any compact set. Given any compact subset $F \subset \mathbb{R}_{\geq 0}^{n}$ containing the set $P$, there exists a class $\mathcal{K}_{\infty}$ function, $\alpha=\alpha_{P, F}$, such that

$$
\Psi(x, \bar{x}) \geq \alpha(|x-\bar{x}|)
$$

$\forall \bar{x} \in P, x \in F \cap \mathcal{S}_{\bar{x}}$.

Proof. Pick any compact set $P \subset \mathcal{E}$. Let $F \subset \mathbb{R}_{\geq 0}^{n}$ be any compact set which contains $P$, and let $R_{0}$ be such that the closed ball $|x| \leq R_{0}$ contains the set $F$. Define $R=R_{0}+\max _{\bar{x} \in P} \bar{x}$. Note that, for every $\bar{x} \in P$, the ball $|x-\bar{x}| \leq R$ also contains the set $F$. Consider the function $\mathbb{R}_{\geq 0}^{n} \rightarrow \mathbb{R}_{\geq 0}^{n}$ given by

$$
\alpha(r):= \begin{cases}\frac{r}{r+1} \min \left\{\Psi(x, \bar{x}): \bar{x} \in P, x \in \mathcal{S}_{\bar{x}}, r \leq|x-\bar{x}| \leq R\right\} & \forall 0 \leq r \leq R, \\ \alpha(R) \frac{r}{R} & \forall r>R .\end{cases}
$$

As discussed above, for $x \in \mathcal{S}_{\bar{x}}, \Psi(x, \bar{x})=0$ if and only if $x=\bar{x}$. Since the minimum is taken over a compact set, the function $\alpha$ satisfies $\alpha(0)=0$ and $\alpha(r)>0$ for $r>0$. Also clearly, for $R \leq r, \alpha$ is strictly increasing and satisfies $\alpha(r) \rightarrow+\infty$ as $r \rightarrow+\infty$. For $0 \leq r \leq R, \alpha(r)$ is also strictly increasing, as a product of a strictly increasing function and a nondecreasing function. By construction, $\Psi(x, \bar{x}) \geq \alpha(|x-\bar{x}|)$ for all $\bar{x} \in P, x \in F \cap \mathcal{S}_{\bar{x}}$. Finally, without loss of generality we may assume that $\alpha$ is continuous on $\mathbb{R}_{\geq 0}$ (otherwise, it is possible to construct a continuous $\tilde{\alpha}, \tilde{\alpha}(0)=0$, with $\alpha(r) \geq \tilde{\alpha}(r)$, and $\tilde{\alpha}(r) \rightarrow+\infty$ as $r \rightarrow+\infty)$.

Pick any $\varepsilon>0$ and consider the sets $\mathbb{U}_{\varepsilon}$ and $\mathcal{W}$ as defined in (3.2) and (3.3), respectively. For any point $\bar{x}_{0} \in \mathcal{E}$, set $V_{0}(x) \equiv V\left(x, \bar{x}_{0}\right)$, where $V$ is the function defined in (5.1). As in section 2, let $\mathcal{S}_{\bar{x}_{0}}$ be the class that contains $\bar{x}_{0}$.

Proposition 6.3. Given any compact sets $P \subset \mathcal{E}$ and $F \subset \mathbb{R}_{\geq 0}^{n}$ containing $P$, there exist class $\mathcal{K}_{\infty}$ functions $\alpha=\alpha_{P, F}$ and $\gamma=\gamma_{P, F}$ such that, for every $\bar{x}_{0} \in$ $P \cap E_{A_{0},+}$ for some $A_{0} \in \mathbb{U}_{\varepsilon}$,

$$
\nabla V_{0}(x) f(x, u) \leq-\alpha\left(\left|x-\bar{x}_{0}\right|\right)+\gamma\left(\left|u-A_{0}\right|_{\mathrm{ecl}}\right)
$$

for every $u \in \mathbb{U}_{\varepsilon}$ and every $x \in F \cap \mathcal{S}_{\bar{x}_{0}} \cap \mathbb{R}_{>0}^{n}$.

Proof. Pick any compact sets $P \subset \mathcal{E}$ and $F \subset \mathbb{R}_{\geq 0}^{n}$ containing $P$. Let $c(P, \varepsilon)$ be the constant given by Lemma 6.1 , and let $\tilde{\alpha}=\tilde{\alpha}_{P, F}$ be the $\mathcal{K}_{\infty}$ function given by 
Lemma 6.2. Now, pick any $\bar{x}_{0} \in P \cap E_{A_{0},+}$ for some $A_{0}=\left(a_{i j}^{0}\right) \in \mathbb{U}_{\varepsilon}$. Using the notation $q_{i} \equiv q_{i}\left(x, \bar{x}_{0}\right)$ (defined in $\left.(5.4)\right)$, we have

$$
\begin{aligned}
\nabla V_{0}(x) f(x, u)= & \sum_{i=1}^{m} \sum_{j=1}^{m} u_{i j} e^{\left\langle b_{j}, \rho\left(\bar{x}_{0}\right)\right\rangle} e^{q_{j}}\left(q_{i}-q_{j}\right) \\
= & \sum_{i=1}^{m} \sum_{j=1}^{m} u_{i j} e^{\left\langle b_{j}, \rho\left(\bar{x}_{0}\right)\right\rangle} e^{q_{j}}\left(q_{i}-q_{j}-\left(e^{q_{i}-q_{j}}-1\right)\right) \\
& +\sum_{i=1}^{m} \sum_{j=1}^{m} u_{i j} e^{\left\langle b_{j}, \rho\left(\bar{x}_{0}\right)\right\rangle} e^{q_{j}}\left(e^{q_{i}-q_{j}}-1\right) \\
= & -\sum_{i=1}^{m} \sum_{j=1}^{m} u_{i j} e^{\left\langle b_{j}, \rho\left(\bar{x}_{0}\right)\right\rangle} e^{q_{j}} \omega\left(q_{i}-q_{j}\right) \\
& +\sum_{i=1}^{m} \sum_{j=1}^{m}\left(u_{i j}-a_{i j}^{0}\right) e^{\left\langle b_{j}, \rho\left(\bar{x}_{0}\right)\right\rangle}\left(e^{q_{i}}-e^{q_{j}}\right),
\end{aligned}
$$

where $\omega(r)$ is the function defined in (5.5). The last equality is justified because $\omega(r) \geq 0 \forall r \in \mathbb{R}$ and, by (5.7),

$$
\sum_{i=1}^{m} \sum_{j=1}^{m} a_{i j}^{0} e^{\left\langle b_{j}, \rho\left(\bar{x}_{0}\right)\right\rangle}\left(e^{q_{i}}-e^{q_{j}}\right)=\left(e^{q_{1}}, \ldots, e^{q_{m}}\right)^{\prime} \tilde{A}_{0} \theta_{B}\left(\bar{x}_{0}\right)=0 .
$$

Applying Lemmas 6.1 and 6.2 , there is a $\mathcal{K}_{\infty}$ function $\tilde{\alpha}$ such that

$$
\begin{aligned}
\nabla V_{0}(x) f(x, u) \leq & -c(P, \varepsilon) \tilde{\alpha}\left(\left|x-\bar{x}_{0}\right|\right) \\
& +\left|u-A_{0}\right|_{\text {ec }} \sum_{i=1}^{m} \sum_{j=1}^{m} e^{\left\langle b_{j}, \rho\left(\bar{x}_{0}\right)\right\rangle}\left|e^{q_{i}}-e^{q_{j}}\right| .
\end{aligned}
$$

Next, let

$$
c_{2}(P, F)=\left(m^{2}-m\right) \max _{j} \max _{\bar{x}_{0} \in P} e^{\left\langle b_{j}, \rho\left(\bar{x}_{0}\right)\right\rangle} \max _{j} \max _{x \in F} e^{q_{j}}
$$

and observe that

$$
\left|u-A_{0}\right|_{\mathrm{ec}} \sum_{i=1}^{m} \sum_{j=1}^{m} e^{\left\langle b_{j}, \rho\left(\bar{x}_{0}\right)\right\rangle}\left|e^{q_{i}}-e^{q_{j}}\right| \leq 2 c_{2}(P, F)\left|u-A_{0}\right|_{\mathrm{ec} 1}
$$

$\forall x \in F$.

Finally, choose $\alpha=\alpha_{P, F}$ to be $\alpha(r)=c(P, \varepsilon) \tilde{\alpha}(r)$ and $\gamma=\gamma_{P, F}$ to be $\gamma(r)=$ $2 c_{2}(P, F) r$.

6.1. Proof of Theorem 3.5. Let $\varepsilon>0$ be any constant and consider the inputvalue set $\mathbb{U}_{\varepsilon}$ defined in (3.2) and the set $\mathcal{W}$ defined in (3.3). Proposition 5.3 shows that system (2.3) is $\mathbb{R}_{>0}^{n}$-forward complete with respect to input maps in $\mathcal{W}$.

Choose any compact sets $P \subset \mathcal{E}$ and $F \subset \mathbb{R}_{\geq 0}^{n}$ with $P \subset F$. Pick any element $\bar{x}_{0} \in P$ and any matrix $A_{0} \in \mathbb{U}_{\varepsilon}$ so that $\bar{x}_{0}=\bar{x}\left(x_{0}, A_{0}\right)$ for some $x_{0} \in F \cap \mathbb{R}_{>0}^{n}{ }^{2}$ Using

\footnotetext{
${ }^{2}$ If no such $A_{0}$ exists, that is, if $P \cap E_{A_{0},+}=\emptyset \forall A_{0} \in \mathbb{U}_{\varepsilon}$, then there is nothing to prove, because the statement of Definition 3.2 is vacuous. But if $A_{0}$ exists, then $x_{0}$ always exists, for instance, $\bar{x}_{0}$.
} 
this element $\bar{x}_{0}$, construct the function $V_{0}(x):=V\left(x, \bar{x}_{0}\right)$. This function $V_{0}$ satisfies properties (i) and (ii) of Definition 3.3 and Proposition 6.3 establishes property (iii). Hence $V_{0}$ is a uniformly semiglobal ISS-Lyapunov function for system (2.3).

By Lemma 3.4, it follows that system (2.3) is uniformly semiglobal ISS with the input-value set $\mathbb{U}_{\varepsilon}$, as we wanted to show.

6.2. Proof of Theorem 3.6. Let $\varepsilon>0$ be any constant and consider the input value set $\mathbb{U}_{\varepsilon}$ defined in (3.2) and the set $\mathcal{W}$ defined in (3.3). Proposition 5.3 shows that system (2.3) is $\mathbb{R}_{>0}^{n}$-forward complete with respect to input maps in $\mathcal{W}$. Assume that system (2.3) is mass conservative, i.e., there exists $v=\left(v_{1}, \ldots, v_{n}\right)^{\prime} \in \mathbb{R}_{>0}^{n}$ so that $\langle v, f(x, u)\rangle=0$ for every $x \in \mathbb{R}^{n}$ and every $u \in \mathbb{U}_{\varepsilon}$.

Choose any compact subset $P \subset \mathcal{E}$ and put

$$
F(P):=\text { closure }\left\{q \in \mathbb{R}_{>0}^{n}: \bar{x}(q, A) \in P \text { for some } A \in \mathbb{U}_{\varepsilon}\right\} .
$$

Then $F(P)$ is a compact subset of $\mathbb{R}_{\geq 0}^{n}$ because it is closed and also bounded, since

$$
q \in F(P) \Rightarrow \exists \bar{x} \in P \text { such that } v_{i} q_{i} \leq\langle v, q\rangle=\langle v, \bar{x}\rangle \Rightarrow q_{i} \leq \frac{1}{v_{i}}|v||\bar{x}| \leq \bar{c} \frac{|v|}{v_{i}}
$$

$\forall i$, where $\bar{c}=\max \{|\bar{x}|: \bar{x} \in P\}$. Moreover, given any $\bar{x} \in P, F(P)$ contains the whole class $\mathcal{S}_{\bar{x}}$.

Now, pick any element $\bar{x}_{0} \in P$ and any matrix $A_{0} \in \mathbb{U}_{\varepsilon}$ so that $\bar{x}_{0}=\bar{x}\left(x_{0}, A_{0}\right)$ for some $x_{0} \in F(P) \cap \mathbb{R}_{>0}^{n} .{ }^{2}$ Using this element $\bar{x}_{0}$, construct the function $V_{0}(x):=$ $V\left(x, \bar{x}_{0}\right)$. This function $V_{0}$ satisfies properties (i) and (ii) of Definition 3.3. Moreover, the two $\mathcal{K}_{\infty}$ functions provided by Proposition 6.3 depend only on $P$ :

$$
\alpha=\alpha_{P, F(P)} \equiv \alpha_{P} \quad \text { and } \quad \gamma=\gamma_{P, F(P)} \equiv \gamma_{P} .
$$

So, $V_{0}$ is in fact a uniformly ISS-Lyapunov function for system (2.3).

By Lemma 3.4, it follows that system (2.3) (when constrained to take input maps in $\mathcal{W})$ is uniformly ISS with the input-value set $\mathbb{U}_{\varepsilon}$.

7. Conclusions. We have extended the analysis of zero deficiency biochemical networks to the case where the kinetic parameters associated with each reaction rate are assumed to be time-varying inputs. We have shown that these rate controlled biochemical systems are input-to-state stable with respect to an appropriate input set. Thus one may analyze the stability of the biochemical network when the reaction rates are controlled by some independent process; for instance, some reactions may be inhibited or activated through enzymatic activity. Also as a consequence of the ISS property, we conclude that such systems of biochemical networks are robust with respect to small perturbations in the kinetic parameters such as temperature fluctuations.

By definition, the zero deficiency biochemical networks are assumed to be closed systems in the sense that there are no inflows or outflows (such as additive inputs). While the systems we have studied also do not allow any inflows or outflows, we have nevertheless incorporated outside effects, in the form of "multiplicative" inputs, by allowing an independent system to control the reaction rates.

Appendix. The Perron eigenvector $\mathbf{v}_{\mathbf{P}}$. By a rational function everywhere defined on $\mathcal{G}$ we mean a function $\psi: \mathcal{G} \rightarrow \mathbb{R}^{m}$ for which every coordinate is a quotient $\psi_{i}=p_{\text {num }} p_{\text {den }}^{-1}$ of two polynomial functions (on the entries of $G$ ) $p_{\text {num }}, p_{\text {den }}: \mathbb{R}^{m \times m} \rightarrow \mathbb{R}$ such that $p_{\text {den }}(G) \neq 0 \forall G \in \mathcal{G}$. 
Proposition A.1. The map $v_{P}$ is a rational function on $\mathcal{G}$.

Proof. For each $G \in \mathcal{G}$, by an abuse of notation, we write $v_{\mathrm{P}}$ for $v_{\mathrm{P}}(G)$. We will also drop the subscript and we let $M=M_{G}$ for simplicity. We have $M v_{\mathrm{P}}=$ $\sigma(M) v_{\mathrm{P}} \Leftrightarrow(M-I) v_{\mathrm{P}}=0$. The matrix $M-I$ has rank $m-1$ because $\sigma(M)=1$ is a simple root of the characteristic polynomial of $M$. Put $M-I=\left(N_{1} N\right)$, where $N_{1}$ is the first column of $M-I$ and $N$ is the remaining $m \times(m-1)$ matrix, and notice that

$$
\left(N_{1} N\right)\left(\begin{array}{c}
1 \\
w_{\mathrm{P}}
\end{array}\right)=0 \Leftrightarrow N w_{\mathrm{P}}=-N_{1} .
$$

Claim. The matrix $N$ has full rank.

Suppose the claim is false. Then there exists an element $u$ in the kernel of $N$, and one can write $N\left(w_{\mathrm{P}}+u\right)=-N_{1}$. But if this is true, then it also holds that

$$
(M-I)\left(\begin{array}{c}
1 \\
w_{\mathrm{P}}+u
\end{array}\right)=0
$$

which implies $w_{\mathrm{P}}+u=w_{\mathrm{P}}$, because $v_{\mathrm{P}}$ is in fact the unique vector with first coordinate equal to 1 in the kernel of $M-I$. So $u \equiv 0$, which proves the claim.

It follows that $\operatorname{det}\left(N^{\prime} N\right) \neq 0$ for every $G$, and applying the Moore-Penrose pseudo-inverse of $N$ yields

$$
v_{\mathrm{P}}=\left(\begin{array}{c}
1 \\
w_{\mathrm{P}}
\end{array}\right)=\left(\begin{array}{c}
1 \\
-\left(N^{\prime} N\right)^{-1} N^{\prime} N_{1}
\end{array}\right),
$$

where $N$ and $N_{1}$ are defined from $M=M_{G}$, as above. This shows that $v_{\mathrm{P}}$ is a rational function on $\mathcal{G}$.

For every $G \in \mathcal{G}$, the Perron eigenvector of $M_{G}, v_{\mathrm{P}}$, is also an eigenvector of the matrix $G$, corresponding to the 0 eigenvalue, and has multiplicity 1 . This fact follows from two observations.

1. $\operatorname{ker}(G) \neq \emptyset$, so $\exists v \in \mathbb{R}^{m} \backslash\{0\}$ such that $G v=0$.

This is because $\overline{1} G=0$, which means that the rows of $G$ are linearly dependent and thus have $\operatorname{rank} G \leq m-1$.

2. Any $v$ such that $G v=0$ satisfies $v \in \operatorname{span}\left\{v_{\mathrm{P}}\right\}$.

This follows from

$$
(\phi(G) G+I) v=v \Rightarrow(\phi(G) G+I)^{m-1} v=M_{G} v=v,
$$

and hence $v \in \operatorname{span}\left\{v_{\mathrm{P}}\right\}$, since $\sigma\left(M_{G}\right)=1$ is an eigenvalue of $M_{G}$, of multiplicity 1 .

Therefore, the kernel of $G$ has dimension 1 and is given by

$$
\operatorname{ker}(G)=\operatorname{span}\left\{v_{\mathrm{P}}(G)\right\} .
$$

Acknowledgment. The author would like to thank Eduardo Sontag for many helpful discussions and suggestions.

\section{REFERENCES}

[1] P.W. Atkins, Physical Chemistry, 5th ed., Oxford University Press, Oxford, 1994.

[2] M. Arcak, D. Angeli, and E.D. Sontag, A unifying integral ISS framework for stability of nonlinear cascades, SIAM J. Control Optim., 40 (2002), pp. 1888-1904. 
[3] R.P. Bywater, A. Sørensen, P. Røgen, And P.G. HJorth, Construction of the simplest model to explain complex receptor activation kinetics, J. Theoret. Biol., 218 (2002), pp. 139-147.

[4] M. Chaves And E.D. Sontag, State-estimators for chemical reaction networks of FeinbergHorn-Jackson zero-deficiency type, Eur. J. Control, 8 (2002), pp. 343-359.

[5] M. Chaves, E.D. Sontag, And R. Dinerstein, Steady-states of receptor-ligand dynamics: A theoretical framework, J. Theoret. Biol., 227 (2003), pp. 413-428.

[6] M. Feinberg, Mathematical aspects of mass action kinetics, in Chemical Reactor Theory: A Review, L. Lapidus and N. Amundson, eds., Prentice-Hall, Englewood Cliffs, NJ, 1977, pp. $1-78$.

[7] M. Feinberg, Chemical reaction network structure and the stabiliy of complex isothermal reactors - I. The deficiency zero and deficiency one theorems, Chem. Engrg. Sci., 42 (1987), pp. 2229-2268.

[8] M. Feinberg, The existence and uniqueness of steady-states for a class of chemical reaction networks, Arch. Ration. Mech. Anal., 132 (1995), pp. 311-370.

[9] F.J.M. Horn and R. Jackson, General mass action kinetics, Arch. Ration. Mech. Anal., 49 (1972), pp. 81-116.

[10] R.A. Horn and C.R. Johnson, Matrix Analysis, Cambridge University Press, New York, 1999.

[11] M. Krichman, E.D. Sontag, And Y. WAng, Input-output-to-state stability, SIAM J. Control Optim., 39 (2001), pp. 1874-1928.

[12] D.A. Lauffenburger and J.J. Linderman, Receptors: Models for Binding, Trafficking, and Signaling, Oxford University Press, New York, 1993.

[13] T.W. McKeithan, Kinetic proofreading in T-cell receptor signal transduction, Proc. Natl. Acad. Sci. USA, 92 (1995), pp. 5042-5046.

[14] D. Siegel and D. MacLean, Global stability of complex balanced mechanisms, J. Math. Chem., 27 (2000), pp. 89-110.

[15] E.D. Sontag, Smooth stabilization implies coprime factorization, IEEE Trans. Automat. Control, 34 (1989), pp. 435-443.

[16] E.D. Sontag, Mathematical Control Theory: Deterministic Finite Dimensional Systems, 2nd ed., Springer-Verlag, New York, 1998.

[17] E.D. SonTAg, Structure and stability of certain chemical networks and applications to the kinetic proofreading model of T-cell receptor signal transduction, IEEE Trans. Automat. Control, 46 (2001), pp. 1028-1047. Errata in IEEE Trans. Automat. Control, 47 (2002), p. 705 .

[18] E.D. Sontag And Y. WANG, On characterizations of the input-to-state stability property, Systems Control Lett., 24 (1995), pp. 351-359.

[19] P.J. Woolf, T.P. Kenakin, And J.J. Linderman, Uncovering biases in high throughput screens of G-protein coupled receptors, J. Theoret. Biol., 208 (2001), pp. 403-418. 